\title{
CLASSES OF SEQUENCES OF POSITIVE NUMBERS
}

\author{
E. H. MOORE 1
}

The results presented in the following pages engaged the attention of Professor E. H. Moore at various times, but were never written up for publication. Notes dated December 1909 indicate that the impetus came in connection with utilization of the "no last absolutely convergent series" theorem of Du Bois-Reymond (see General analysis, p. 48) and the paper of Landau in Nachr. Ges. Wiss. Göttingen (1907) pp. 25-27) in which Landau proves that if $\left\{a_{n}\right\}$ is a sequence such that $\sum\left|a_{n} b_{n}\right|$ converges for all sequences such that $\sum\left|b_{n}\right|{ }^{p}$, with $p>1$, converges, then $\sum\left|a_{n}\right|^{p /(p-1)}$ converges. This latter theorem proved interesting not only because it is a sort of converse to the theorem: If $\sum\left|a_{n}\right|^{p}$ converges and if $\sum\left|b_{n}\right|^{p /(p-1)}$ converges then $\sum\left|a_{n} b_{n}\right|$ converges, a consequence of Hoelder's inequality, but also because the theorem which Landau actually proved was the contrapositive equivalent theorem, namely, if $\sum\left|a_{n}\right|^{p /(p-1)}$ diverges, then there exists a sequence such that $\sum\left|b_{n}\right|^{p}$ converges, but $\sum\left|a_{n} b_{n}\right|$ diverges. By noting the trivial identity $a_{n}=\left(a_{n} b_{n}\right)\left(1 / b_{n}\right)$, the last theorem has the skeleton form: If the sequence $\left\{a_{n}\right\}$ belongs to a class $\mathfrak{M}_{1}$ (namely, the divergent $p /(p-1)$ power) then there exist two sequences $\left\{b_{n}\right\}$ and $\left\{c_{n}\right\}$ such that $a_{n}=b_{n} c_{n}$, and $\left\{b_{n}\right\}$ belongs to $\mathfrak{M}_{2}$ (convergent $p$ the power) and $\left\{c_{n}\right\}$ to $\mathfrak{M}_{3}$ (divergent). Since logical questions were always of great interest to him, this instance in which the contrapositive of a given theorem has an independently interesting statement led him to speculations concerning the true nature of contrapositive proof. Among other things he stressed the idea that many so-called contrapositive proofs could be formulated to advantage as direct proofs.

From his notes, it appears that the subject matter of the first half of this paper engaged his continued attention from December 1910 to January 1911. But apparently he made no attempt to write up the results for publication until about 1918. However, only a few sections of the manuscript were completed. On several occasions he worked out a "multiplication table" (see end of Part II) and added to it from time to time. We have not always been able to connect the references which some of these tables contain with his notes, and consequently reconstruct his proofs. Instead we have used the final re-

Received by the editors July 11,1945 .

${ }^{1}$ Prepared for publication by H. H. Goldstine and T. H. Hildebrandt. 
sults and taken the liberty of arranging the proofs in what appears to us as a possible logical order.

The first part of the paper, which serves as an introduction, gives the basic notation and certain general theorems and their interrelations (most of which are taken from the unfinished manuscript). In the second part of the paper, we give a body of theorems sufficient to cover all of the cases of the "multiplication table" which concerns itself with a system of classes of sequences of positive numbers, and is in a sense closed in this system. The third part of the paper is concerned with what Professor Moore called a General Multiplication Theorem, which he stated in a letter to T. H. Hildebrandt dated January 4, 1926, but gave no hint as to its proof. Since no proof appears in his notes, we have worked out a proof of the theorem in question.

Whether Professor Moore would have approved of the publication of these results, since in many ways they seem trivial, we do not know. We have found some of these things interesting to play with and it is not impossible that they may suggest further results which are worth while. The connection with linear functional operations on sequences, the closure of the multiplication table, and the fact mentioned in footnote 5 that the Hoelder inequality basic to the Pringsheim-Landau Theorem, and the proof of the Abel-Dini Theorem basic to the Landau Theorem, both can be derived from the same inequality: $x^{e} \leqq 1+e(x-1)$ for $0<e<1, x>0$, are some of the points which made these developments attractive to us.

H. H. Goldstine

T. H. HILDEBRANDT

\section{INTRODUCTION}

1.1. The class $V$. We consider throughout sequences of positivenumbers: $\alpha=\left\{a_{n}\right\}$, with $a_{n}>0$. Such sequences may be regarded as positive-valued functions on the range $\mathfrak{P}^{\mathrm{III}}$ of positive integers, or geometrically as points in the space of infinite dimensions, limited however to points all of whose coordinates are positive. We designate the space of all such sequences by $V$ and call its elements either sequences, functions or points. Since we shall be interested only in elements of $V$, it will be understood that the word sequence refers only to a sequence consisting of positive terms.

We shall be interested in certain subspaces or subclasses of $V$, the points of each class being all of the points of $V$ which possess a certain property. For convenience we use the same notation for the class and the characteristic property, that is, the class $P$ is the class of all sequences having the property $P$. We shall use the standard notation 
relative to sum and product of classes, that is, $P \cup Q$, the sum of $P$ and $Q$, or the totality of all sequences which have either the property $P$ or the property $Q, P \cap Q$, the product of $P$ and $Q$, or all sequences having both the property $P$ and $Q .-P$ shall be the complement of $P$ relative to $V$, that is, the class of sequences not having the property $P$.

In an obvious way we say that a class $P$ contains a class $Q$ if every element of $Q$ is contained in $P$. With respect to properties, $Q$ implies $P$ if the class $P$ contains $Q$. Under the circumstances we can speak of a monotonically increasing or decreasing set of classes.

1.2. Fundamental subclasses of $V$. We consider the following basic classes (properties):

$F$ : the class of all bounded sequences, that is, of all points in which the least upper bound of the coordinates is finite;

$L_{0}$ : the class of all sequences having zero as limit;

$L_{\infty}$ : the class of all sequences having infinity as limit;

$C$ : the class of all sequences with convergent sum;

$D:$ the class of all sequences with divergent sum.

Obviously $C=-D$.

Any sequence and real number $p$ defines the sequence $\alpha^{p}=\left\{a_{n}^{p}\right\}$. For every property $P$ and number $p$, the property $P_{p}$ of a point $\alpha$ is defined as the property $P$ of $\alpha^{p}$. Thus $C_{p}$ is the class of all sequences such that $\sum a_{n}^{p}$ is convergent; $D_{p}$ is defined in a similar way.

We note that if $0<e<f$, then the class $C_{f}$ properly contains the class $C_{e}, C_{-f}$ properly contains $C_{-e}, D_{f}$ is contained in $D_{e}$ and $D_{-f}$ is contained in $D_{-\varepsilon}$. Obviously if $e>0$, then $F$ and $F_{e}, L_{0}$ and $\left(L_{0}\right)_{e}, L_{\infty}$ and $\left(L_{\infty}\right)_{e}$ are each identical. But $\left(L_{0}\right)_{-c}=\left(L_{0}\right)_{-1}$ is $L_{\infty}$ and $\left(L_{\infty}\right)_{-e}$ $=\left(L_{\infty}\right)_{-1}$ is $L_{0} . F_{-6}$ is the same as $F_{-1}$, which is the set of sequences such that lim $\sup _{n}\left(1 / a_{n}\right)<\infty$, that is, the class of sequences bounded from zero. Then $-F_{-1}$ is the class such that $\lim \inf _{n} a_{n}=0$.

1.3. Derived classes. If for $e>0$, the system of classes $P_{e}$ is monotonic increasing in $e$, then we define

$$
P_{!}^{\prime}=\bigcup_{0<f<0} P_{f} \text { and } P_{c}^{\prime \prime}=\bigcap_{f>0} P_{f}
$$

Thus $C_{\theta}^{\prime}=\mathrm{U}_{0<f<{ }^{\circ}} C_{f}$ is the class of sequences $\alpha$ such that there exists an $f<e$ such that $\alpha^{f}$ is in $C . C_{e}^{\prime \prime}$ is the class of sequences such that $\alpha^{f}$ is in $C$ for all $f>e$.

If for $e>0$ the system of classes $P_{e}$ is monotonic decreasing in $e$, then the inequalities in the definitions are reversed, that is, we define

$$
P_{a}^{\prime}=\bigcup_{>\rightarrow e} P_{f} \text { and } P_{a}^{\prime \prime}=\bigcap_{0<f<e} P_{f} .
$$


This applies then to the definitions of $D_{e}^{\prime}$ and $D_{e}^{\prime \prime}$ as well as $D_{-, e}^{\prime}$ and $D_{-e}^{\prime \prime}$ with $e>0$. In general $P^{\prime}$ is a sum of classes, $P^{\prime \prime}$ is a product of classes.

The application of these notions to the limiting cases $e=0, \infty$, is obvious, but one can define only the following:

$C_{0}^{\prime \prime}$ : for every $e$ the property $C_{e} ; \quad C_{-0}^{\prime \prime}$ : for every $e$ the property $C_{-\varepsilon}$;

$C_{\infty}^{\prime}$ : for some $e$ the property $C_{\theta} ; \quad C_{-\infty}^{\prime}$ : for some $e$ the property $C_{-\theta}$;

$D_{0}^{\prime}$ : for some $e$ the property $D_{e} ; \quad D_{-0}^{\prime}$ : for some $e$ the property $D_{-e}$;

$D_{\infty}^{\prime \prime}$ : for every $e$ the property $D_{e} ; \quad D_{-\infty}^{\prime \prime}$ : for every $e$ the property $D_{-e}$.

An application of the same process to the classes $C^{\prime}$ and $D^{\prime}$ yields nothing new in classes, for we have the following results for $e>0$ :

(a) $\underset{0<f<e}{\bigcup} C_{f}^{\prime}=C_{0}^{\prime}$;

(c) $\underset{0<j<e}{\bigcup} C_{f}^{\prime \prime}=C_{e}^{\prime}$;

$$
\begin{aligned}
& \bigcap_{>0} C_{f}^{\prime}=C_{!^{\prime \prime} ;} \\
& \bigcap_{f>0} C_{f}^{\prime \prime}=C_{!^{\prime \prime} ;}
\end{aligned}
$$

and similar results for classes of divergent series and negative exponents. Of these (a) and (d) are obvious. For (b), suppose $\alpha$ belongs to $C_{a}^{\prime \prime}$. Then $\alpha$ is of $C_{f}$ for all $f>e$. Consequently $\alpha$ belongs to $C_{o}^{\prime}$ for all $g>f>e$, that is, for all $g>e$. Conversely, suppose that $\alpha$ belongs to $\cap_{>>e} C_{f}^{\prime}$. Then $\alpha$ is of $C_{f}^{\prime}$ for all $f>e$, that is, there exists a $g<f$, such that $\alpha$ is of $C_{g}$. As a consequence $\alpha$ is of $C_{h}$ for all $h>g>e$, that is, $\alpha$ is of $C_{\theta}^{\prime \prime}$. Similar considerations show the validity of (c). In a way the classes $P^{\prime}$ are of the nature of greatest of limits of a set of classes, and $P^{\prime \prime}$ of the least of limits, as these results seem to indicate.

1.4. Fundamental systems of subclasses of $V$. We shall be interested in the following two systems of subclasses of $V$ :

\section{System I}

$$
\begin{gathered}
0, C_{0}^{\prime \prime}, C_{e}^{\prime}, C_{e}, C_{e}^{\prime \prime}, C_{f}^{\prime}, C_{f}, C_{f}^{\prime \prime}, C_{\infty}^{\prime}, L_{0}, F \cap-F_{-1}, F \text { or }-F_{-1}, \\
F \cup-F_{-1},-L_{\infty}, D_{-\infty}^{\prime \prime}, D_{-f}^{\prime}, D_{-f}, D_{-f}^{\prime \prime}, D_{-\infty}^{\prime}, D_{-\bullet}, D_{-\infty}^{\prime \prime}, D_{-0,}^{\prime}, V .
\end{gathered}
$$

\section{System II}

$$
\begin{gathered}
V, D_{0}^{\prime}, D_{\bullet}^{\prime \prime}, D_{\bullet}, D_{\bullet}^{\prime}, D_{f}^{\prime \prime}, D_{f}, D_{f}^{\prime}, D_{\infty}^{\prime \prime},-L_{0},-F \cup F_{-1},-F \text { or } F_{-1}, \\
-F \cap F_{-1}, L_{\infty}, C_{-\infty}^{\prime}, C_{-f}^{\prime \prime}, C_{-f}, C_{-f}^{\prime}, C_{-\bullet,}^{\prime \prime}, C_{-e,}, C_{-e}^{\prime}, C_{-0,0}^{\prime \prime},
\end{gathered}
$$

In the first system, the classes are of increasing content, assuming $0<e<f$, each class being a proper subclass of every succeeding class (excepting that there is a certain ambiguity in the middle of each, the remark preceding holding in each system whichever reading is 
adopted). In the second system the classes are complements relative to $V$ of the corresponding classes of the first system, so that the second system is of decreasing content.

The proper monotoneity is in general obvious, excepting for cases of the type: $C_{\theta}^{\prime}<C_{\theta}<C_{\theta}^{\prime \prime}$. For this it is sufficient to consider the case where $e=1$. We note that the sequence $a_{n}=1 / n$ is an element of $C_{0}$ for $e>1$, but not for $e=1$, that is, is of $C_{1}{ }^{\prime \prime}$, but not of $C_{1}$. On the other hand if $a_{n}=1 / n \log ^{2} n$, then $\alpha$ is an element of $C_{1}$ but not of $C_{\theta}$ for $e<1$, that is, of $C_{1}$ but not of $C_{1}^{\prime}$. Since $P<Q$ implies $P_{-1}<Q_{-1}$, we get at once $C_{-\theta}^{\prime}<C_{-\theta}<C_{-e}^{\prime \prime}$. The corresponding results for divergent series follow by taking complements.

1.5. Multiplications of sequences, and classes of sequence multiplications. If $\alpha=\left\{a_{n}\right\}$ and $\beta=\left\{b_{n}\right\}$ are any two sequences of $V$, then $\alpha \times \beta$ is the sequence $\left\{a_{n} b_{n}\right\}$ of $V$.

In a similar manner if $P$ is the class of sequences $\alpha$ having the property $P$ and $Q$ the class of sequences $\beta$ having the property $Q$, then we denote by $P \times Q$ the class of sequences $\alpha \times \beta$ with $\alpha$ in $P$ and $\beta$ in $Q$, that is, $\gamma$ is in $P \times Q$ if and only if there exists an $\alpha$ of $P$ and a $\beta$ of $Q$ such that $\gamma=\alpha \times \beta$. The Pringsheim-Landau Theorem gives a characterization of the classes $P \times Q$, where $P=C_{1 / /}, Q=C_{1 / f}$, and $P \times Q=C_{1 /(\theta+f)}, e>0, f>0$.

By analogy, we define the class $P / Q$ as the class of all elements $\gamma$ such that for all $\beta$ of $Q$, the product sequence $\gamma \times \beta$ is of $P$. The Landau Theorem states a result for the class $P / Q$ for the case where $P=C_{1 /(\theta+f)}$, and $Q$ is $C_{1 / f}$, namely, that $P / Q$ is the class $C_{1 / e}, e>0$, $f>0$; or as a matter of fact, where $P$ is $C_{1}, Q$ is $C_{p}$ and $P / Q$ is $C_{p^{\prime}}$ with $p>1$ and $(1 / p)+\left(1 / p^{\prime}\right)=1$.

The following relations are self-evident:

$1.51 P \times Q=Q \times P ;(P \times Q)_{-1}=P_{-1} \times Q_{-1} ;(P / Q)_{-1}=P_{-1} / Q_{-1}$.

There also exist relations between the product and quotient processes embodied in the following theorem:

$$
P / Q=-\left(Q \times-P_{-1}\right)_{-1},
$$

from which we deduce by an obvious substitution the corollary:

$$
P \times Q=-\left(-P_{-1} / Q\right)_{-1} \text {. }
$$

For the proof suppose that $P / Q=R$, that is, $\gamma$ in $R$ is equivalent to a $\gamma$ such that, for every $\beta$ of $Q$, it is true that $\gamma \times \beta$ is in $P$. Then $\gamma$ in $-R$ is equivalent to a $\gamma$ such that there exists a $\beta$ of $Q$ for which $\gamma \times \beta$ is of $-P$. Let $\gamma \times \beta=\alpha$. Then $\beta \times \alpha^{-1}=\gamma^{-1}$. Then $\gamma$ of $-R_{-1}$ is equivalent to a $\gamma$ such that there exists a $\beta$ of $Q$ and an $\alpha$ of $-P_{-1}$ 
such that $\gamma=\beta \times \alpha$, symbolically:

$$
-R_{-1}=Q \times-P_{-1} \text { and so } R=-\left(Q \times-P_{-1}\right)_{-1} .
$$

The following additional equivalences are now obvious:

$$
\begin{aligned}
P / Q & =-\left(Q \times-P_{-1}\right)_{-1}=-\left(Q_{-1} \times-P\right) \\
& =-\left(-P \times Q_{-1}\right)=-Q_{-1} /-P_{-1} . \\
P \times Q & =-\left(-P_{-1} / Q_{-1}=-\left(-Q_{-1} / P\right)_{-1} .\right.
\end{aligned}
$$

A theorem of the form $P \times Q=R$ or $P / Q=R$ can be divided into parts, namely, $P \times Q \supset R, P \times Q \subset R$, and $P / Q \supset R$ and $P / Q \subset R$ respectively, the theorem being considered as a relation between the classes. A theorem of the form $P \times Q \supset R$ is called a factorization theorem since it asserts that if $\gamma$ is of $R$ then there exists $\alpha$ of $P$ and $\beta$ of $Q$ such that $\alpha \times \beta=\gamma$. A theorem of the form $P \times Q \subset R$ will be called a multiplication theorem, since it asserts that if $\alpha$ is of $P$ and $\beta$ is of $Q$ than $\alpha \times \beta$ is of $R$. A theorem of the form $P / Q \subset R$ will be called a division or Landau theorem, since it asserts that if $\alpha$ is such that for every $\beta$ of $Q$ it is true that $\alpha \times \beta$ is of $P$, then $\alpha$ is of $R$. The theorem $P / Q \supset R$ is really a multiplication theorem since we have:

1.56. $P / Q \supset R$ is equivalent to $R \times Q \subset P$.

For the statement $P / Q \supset R$ is equivalent to the statement that if $\alpha$ is of $R$ then, for every $\beta$ of $Q$, it is true that $\alpha \times \beta$ is of $P$, which is logically equivalent to: if $\alpha$ is of $R$ and $\beta$ is of $Q$ then $\alpha \times \beta$ is of $P$, that is, a multiplication theorem. We have as an immediate corollary:

1.57. $P / Q \supset R$ is equivalent to $P / R \supset Q$.

Since $P / Q$ is the same as $-Q_{-1} /-P_{-1}$, we have also the following: 1.58. $P / Q \supset R$ is equivalent to $-Q_{-1} /-P_{-1} \supset R$ is equivalent to $-R_{-1} /-P_{-1} \supset Q$ is equivalent to $-Q_{-1} / R_{-} P_{-1}$ is equivalent to $-R_{-1} / Q \supset-P_{-1}$.

The theorem and corollary also give:

1.59. $P \times Q \subset R$ is equivalent to $P \times-R_{-1} \subset-Q_{-1}$ is equivalent to $-R_{-1} \times Q \subset-P_{-1}$.

We note however that the theorem and corollary are not in general true if the implications are reversed, that is, we have:

1.510. $P / Q \subset R$ does not always imply $Q \times R \supset P$.

1.511. $Q \times R \supset P$ does not always imply $P / Q \subset R$.

1.512. $P / Q \subset R$ does not always imply $P / R \subset Q$.

Instances of these are the following, which depend on theorems to be proved later:

ad 1.510. Take $P=L_{0}, Q=C_{1}, R=F$. Then by 2.43

$$
P / Q=L_{0} / C_{1}=-\left(C_{1} \times-L_{\infty}\right)_{-1}=F \text {. }
$$


On the other hand by 2.41

$$
Q \times R=C_{1} \times F=C_{1},
$$

and $C_{1}$ does not contain $L_{0}=P$.

ad 1.511. Take $Q=C_{1}, R=L_{0}$, and $P=C_{1}$. Then by 2.44

$$
Q \times R=C_{1} \times L_{0}=C_{1} \text {, }
$$

but by 2.21

$$
P / Q=C_{1} / C_{1}=-\left(C_{1} \times-C_{-1}\right)_{-1}=-\left(C_{1} \times D_{-1}\right)_{-1}=F
$$

and $F$ is not contained in $R=L_{0}$.

ad 1.512 Take $P=L_{0}, Q=C$ and $R=F$. Then as in $1.510, P / Q \subset R$, but by 2.41

$$
P / R=L_{0} / F=-\left(F \times-L_{\infty}\right)_{-1}=L_{0}
$$

and $L_{0}$ is not contained in $C$.

Other instances of 1.510 and 1.512 are obtained if $P=V, Q=0$ and $R=V$.

Even so, every division or Landau theorem is equivalent to a factorization theorem, and conversely, but the equivalence is not as simple as one might expect. We have:

1.513. The statement $P / Q \subset R$ is equivalent to the statement $Q \times-P_{-1}$ $\supset-R_{-1}$.

This follows immediately from 1.52

In view of these theorems, we shall in the sequel focus primary attention on the product class $P \times Q$, of two classes $P$ and $Q$. We emphasize however that while a theorem of the form $P / Q \supset R$ has as its equivalent the form $Q \times R \subset P$, a theorem of the form $P / Q \subset R$ has the more complicated equivalent $Q \times-P_{-1} \supset-R_{-1}$.

1.6. Set addition and set product. We consider next the effect of set addition and set product on the theorems of multiplication and factorization type. We have the following theorems.

1.61. THEOREM. If $P_{p}$ and $R_{p}$ are any systems of classes such that for every $p: P_{p} \times Q \supset R_{p}$, then $\left(\bigcup P_{p}\right) \times Q \supset \cup R_{p}$. If for every $p: P_{p} \times Q \subset R_{p}$, then $\left(\cup P_{p}\right) \times Q \subset \cup R_{p}$. Consequently if $P_{p} \times Q=R_{p}$ then $\left(\cup P_{p}\right) \times Q$ $=\bigcup R_{p}$.

1.62. ThEOREM. If for every $p: P_{p} \times Q \subset R_{p}$, then $\left(\cap P_{p}\right) \times Q \subset \cap R_{p}$.

These theorems follow immediately from the definitions. However, conclusions concerning $\left(\cap P_{p}\right) \times Q \supset \cap R_{p}$ as a consequence of $P_{p} \times Q \supset R_{p}$ for every $p$ are not so immediate. Suppose for instance 
that $P_{1} \times Q \supset R_{1}$ and $P_{2} \times Q \supset R_{2}$, and assume $\gamma$ is of $R_{1} \cap R_{2}$. Then there exist $\alpha_{1}$ of $P_{1}$ and $\beta_{1}$ of $Q_{1}$ and $\alpha_{2}$ of $P_{2}$ and $\beta_{2}$ of $Q_{2}$ such that $\gamma=\alpha_{1} \times \beta_{1}=\alpha_{2} \times \beta_{2}$. In order that $\gamma$ be of $\left(P_{1} \cap P_{2}\right) \times Q$, it would be necessary to establish the existence of a single $\beta$ of $Q$ and an $\alpha$ of $P_{1} \cap P_{2}$ such that $\gamma=\alpha \times \beta$. A sufficient condition for this can be obtained as follows. If $Q$ has the dominance property ${ }^{2} \Delta_{1}$ (if $\beta_{1}$ and $\beta_{2}$ are of $Q$ then there exists a $\beta$ of $Q$ such that $\beta \geqq \beta_{1}$ and $\beta \geqq \beta_{2}$ (pointwise)) and if $\alpha=\gamma / \beta \leqq \gamma / \beta_{1}=\alpha_{1}$ and $\alpha=\gamma / \beta \leqq \gamma / \beta_{2}=\alpha_{2}$ were in both $P_{1}$ and $P_{2}$, then $\gamma$ would have the property desired. This would certainly occur if the classes $P_{1}$ and $P_{2}$ had the dominance property $\Delta_{0}^{\prime}$ : if $\alpha_{1}$ is of $P$ and $\alpha \leqq \alpha_{1}$ then $\alpha$ is of $P$. Since the same method of proof would apply to a finite number of classes we can state the theorem:

1.63. Theorem. If $Q$ has the dominance property $\Delta_{1}$ and $P_{1}, \cdots, P_{n}$ have each the dominance property $\Delta_{0}^{\prime}$, and if $P_{i} \times Q \supset R_{i}$ for each $i$, then $\left(\cap P_{i}\right) \times Q \supset \cap R_{i}$.

The case when sequences of classes $\left\{P_{m}\right\}$ and $\left\{R_{m}\right\}$ are involved would obviously require some alteration in these conditions. We replace the property $\Delta_{0}^{\prime}$ by the following modification:

A class $P$ of sequences has the dominance property $\Delta_{0}^{\prime \prime}$ if any sequence $\alpha$ which is ultimately dominated by some sequence $\alpha_{0}$ of $P$ belongs to $P$.

We replace the dominance property $\Delta_{1}$ by one involving a sequence of elements, and define:

A class $Q$ of sequences has the dominance property $K$ if for any sequence of elements $\beta_{1}, \cdots, \beta_{m} \cdots$ of $Q$ there exists an element $\beta_{0}$ of $Q$ so that each $\beta_{m}$ is ultimately dominated by $\beta_{0}$, that is, if $\beta_{m}=\left\{b_{m n}\right\}$ and $\beta_{0}=\left\{b_{0 n}\right\}$, then $\lim \sup _{n} b_{m n} / b_{0 n} \leqq 1$ for all $m$.

An examination of the proof of the simple case above shows that the same method will give the theorem:

1.64. TheOREM. If each of the classes $P_{m}$ has the property $\Delta_{0}^{\prime \prime}$, and $Q$ has the property $K$, and if $P_{m} \times Q \supset R_{m}$, then we have $\left(\cap P_{m}\right) \times Q$ $\supset \cap R_{m}$ and consequently if $P_{m} \times Q=R_{m}$ then $\left(\cap P_{m}\right) \times Q=\cap R_{m}$.

All of the classes in System I have the property $\Delta_{0}^{\prime \prime}$. On the other hand the dominance property $\Delta_{1}$ is not found in the classes $-F_{-1}$, $F \cup-F_{-1}, F \cap-F_{-1},-L_{\infty}, D_{-6}$. These do not have the additive property either. For instance, for $D_{-1}$, if $a_{n}=n^{2}$ for $n$ even and $n$ for $n$

${ }^{2}$ For dominance properties see E. H. Moore, Introduction to a form of general analysis, Amer. Math. Soc. Colloquium Publications, vol. 2, New York, 1910, pp. 3941. Since we have used the letter $D$ to signify divergence, we shall use the corresponding Greek letter $\Delta$ for dominance properties. 
odd, while $b_{n}=n$ for $n$ even and $n^{2}$ for $n$ odd, then $\alpha$ and $\beta$ belong to $D_{-1}$ but $\alpha+\beta$ does not, ${ }^{3}$ and $\alpha$ and $\beta$ do not have a common dominant in $D_{-1}$.

The dominance property $K$ is obviously implied by the dominance property $K_{0}$, which is defined as follows: For any sequence of sequences $\beta_{m}$ of $Q$ there exists a sequence $\beta_{0}$ such that $\lim _{n} b_{m n} / b_{0 n}=0$ for every $m$. The classes $V, C_{e}$ and $L_{0}$ have the property $K_{0}$ and consequently $K$, as demonstrated in Introduction to a form of general analysis, pp. $44 \mathrm{ff}$., but since $D_{-e}$ does not have the property $\Delta_{1}$, even in the ultimate sense, $D_{-e}$ does not have the property $K$.

For purposes of reference, we mention the following theorem whose proof is obvious:

1.65. ThEOREM. If $P_{1} \subset P_{2}$, then $P_{1} \times Q \subset P_{2} \times Q$. Consequently if $P_{1} \times Q=P_{2} \times Q=R$, and if $P_{1} \subset P \subset P_{2}$, then $P \times Q=R$.

\section{The Classes $P \times Q$ in System I}

In this part we shall prove theorems sufficient to characterize the class $P \times Q$ where $P$ and $Q$ are any two classes in the System I of 1.2. In view of Theorems $1.52,1.53$ and 1.54 this will give not only a complete set of multiplication and factorization theorems in this system, but also division or Landau theorems. We note that corresponding theorems for the classes of System II are immediate consequences of those in System I, since when $P$ is in I then $P_{-1}$ is in II, and $P \times Q=R$ implies $P_{-1} \times Q_{-1}=R_{-1}$.

2.1. We note in the first place:

\subsection{Theorem I. $-F_{-1} \times-F_{-1}=V$.}

Since the multiplication of any two sequences is a sequence, it is only necessary to prove that if $\gamma=\left\{c_{n}\right\}$ is any sequence, then there exist two sequences $\alpha=\left\{a_{n}\right\}$ and $\beta=\left\{b_{n}\right\}$ such that $\lim \inf a_{n}=0$, and $\lim \inf b_{n}=0$ and $c_{n}=a_{n} b_{n}$. For this purpose we set $a_{n}=1 / n, b_{n}=c_{n} n$ for $n$ even, and $a_{n}=c_{n} n, b_{n}=1 / n$ for $n$ odd.

An immediate consequence of this theorem is:

2.12. $P \times Q=V$ for all $P$ and $Q$ of the system $\mathrm{I}$, such that $-F_{-1} \subseteq P$ $\subseteq V,-F_{-1} \subseteq Q \subseteq V$.

For under these circumstances we have

$$
V=-F_{-1} \times-F_{-1} \subset P \times Q \subset V .
$$

As an instance of this we might note $D_{-\bullet} \times D_{-f}=V$.

\footnotetext{
3 As a matter of fact $D_{-1}+D_{-1}=V$.

4 Cf. Moore, loc. cit. p. 44.
} 


\subsection{Hoelder-Landau theorems.}

2.21. Theorem II. We have (a) $C_{\theta} \times C_{f}=C_{\theta f /(\theta+f)}$ if $e>0, f>0$; (b) $C_{\theta} \times D_{-f}=D_{-e f /(e-f)}$ if $e>f>0$; (c) $C_{e} \times D_{-f}=-F_{-1}$ if $f \geqq e>0$.

The first of these: (a) is mentioned in the introduction. From the inequality:5 $(e+f)(a b)^{1 /(e+f)} \leqq e a^{1 / e}+f b^{1 / f}$ it follows that if $\alpha$ is of $C_{1 / 0}$ and $\beta$ is of $C_{1 / f}$ then $\alpha \times \beta=\gamma$ is of $C_{1 /(e+f)}$. The substitution $e^{\prime}=1 / e$, $f^{\prime}=1 / f, g=1 /(e+f)=e^{\prime} f^{\prime} /\left(e^{\prime}+f^{\prime}\right)$ gives the result $C_{e^{\prime}} \times C_{f^{\prime}} \subset C_{g}$. Conversely suppose $\gamma$ is of $C_{o}$ with $g=e f /(e+f)$. Set $\alpha=\gamma^{g / \theta}$ and $\beta=\gamma^{o / s}$. Then $\alpha$ is of $C_{e}$ and $\beta$ is of $C_{f}$, so that $C_{e} \times C_{f} \supset C_{v}$.

For the second part (b) of this theorem, we use from 1.59 the equivalence of $P \times Q \subset R$ to $P \times-R_{-1} \subset-Q_{-1}$, so that part (a) yields $C_{e} \times D_{-o} \subset D_{-f}$. Setting $g=e f /(e+f)=f^{\prime}$ and $f=g^{\prime}$ gives $g^{\prime}=e f^{\prime} /\left(e-f^{\prime}\right)$, so that we must assume $e>f^{\prime}$, that is, we have, dropping primes, $C_{e} \times D_{-f} \subset D_{-e f /(\theta-f)}$. The converse of this statement, that is, $C_{\varepsilon} \times D_{-f}$ $\supset D_{-o f /(e-f)}$, is the contrapositive of the Landau Theorem. Assume that $\gamma$ is of $D_{-\theta}$, where $g=e f /(e-f)$, and set $\sigma=\left\{s_{n}\right\}=\left\{\sum_{1}^{n} c_{m}^{-0}\right\}$. Then by the Abel-Dini Theorem, ${ }^{6} \gamma^{-\sigma} \times \sigma^{-v}$ is of $C_{1}$ for $v>1$ and of $D_{1}$ for $v \leqq 1$. Consequently if we let $\alpha=\gamma^{-o / e} \times \sigma^{-1 / f}$ and $\alpha \times \beta=\gamma$, that is, $\beta=\gamma^{\sigma / f} \times \sigma^{1 / f}$, then $\alpha^{\varepsilon}=\gamma^{-\theta} \times \sigma^{-e / f}$ which since $e>f$ is of $C_{1}$, so that $\alpha$ is of $C_{\theta}$, while $\beta^{-f}=\gamma^{-\theta} \times \sigma^{-1}$ is of $D_{1}$, that is, $\beta$ is of $D_{-f}$.

Note the similarity in form between the first two parts of this theorem.

For the last part (c) of this theorem, we show first that $C_{\theta} \times D_{-f}$ $C-F_{-1}$, using a contrapositive method of proof. Suppose $\alpha=\left\{a_{n}\right\}$ is of $C_{e}$ and $\beta=\left\{b_{n}\right\}$ is of $D_{-f}$ and if possible lim inf $a_{n} b_{n}>0$. Then there exists an $e_{0}>0$, such that for all $n, a_{n} b_{n}>e_{0}$. Consequently

$$
\sum_{n} a_{n}^{f} \geqq e_{0}^{f}\left(\sum b_{n}^{-f}\right)=\infty,
$$

that is, $\alpha$ is of $D_{f}$ contrary to hypothesis. On the other hand we show that $C_{e} \times D_{-f} \supset-F_{-1}$ for any positive $e$ and $f$. Let $\gamma=\left\{c_{n}\right\}$ be such that $\lim \inf c_{n}=0$, and select a sequence $\alpha_{0}=\left\{a_{0 n}\right\}$ from $C_{e}$. For each $k$ determine $n_{k}>n_{k-1}$ so that $c_{n_{k}}<a_{0 k}$, and define the sequence $\alpha=\left\{a_{n}\right\}$ as follows: $a_{n}=a_{0 n}$ if $n \neq n_{k}, a_{n}=c_{n}$ for $n=n_{k}$. Then obviously:

$$
\sum\left(a_{n}\right)^{\bullet}<2 \sum a_{0 n}^{e}<\infty,
$$

B This inequality can be deduced from the well known inequality: $x^{6} \leqq 1+e(x-1)$, $0 \leqq e \leqq 1, x>0$. Replace $x$ by $A / B$, with $A>0$, and $B>0$. Then $A^{\circ} B^{1-e} \leqq e A+(1-e) B$. Let $e=f /(f+g), f>0, g>0$. Then $1-e=g /(f+g)$. Also set $A^{f}=a$, and $B^{o}=b$. Then the inequality $(f+g)(a b)^{1 /(f+g)} \leqq f a^{1 / f}+g b^{1 / 0}$ results. We might note that $x^{\prime} \geqq 1+e(x-1)$ for $e>1$, and $e<0$, and that each of the three cases can be deduced from each other.

- See for instance Knopp, Infinite series, London, 1928, §39, p. 290. Note that the $C_{1}$ property follows from the initial inequality of the preceding footnote. 
that is, $\alpha$ is of $C_{\theta}$. Obviously $b_{n_{k}}=1$ for all $k$. Hence $\beta=\left\{b_{n}\right\}$ is of $D_{-f}$ for all $f$, or of $D_{-\infty}^{\prime}$.

An examination of the proof just given shows that it applies to a much wider group of classes. In particular we have:

2.22. $P \times Q \supset-F_{-1}$, for any class $P$ contained in $L_{0}$ and any class $Q$ containing $-L_{\infty}$.

From the fundamental Theorem 2.21 we obtain theorems concerning $P \times Q=R$ where $P$ and $Q$ are sums and products of classes of the $C$ or $D_{-1}$ series. The procedure is illustrated in the proof of:

2.23. Theorem III. If $1 / g=1 / e+1 / f$ or $g=e f /(e+f)$, then

$$
C_{e}^{\prime} \times C_{f}=C_{e}^{\prime} \times C_{f}^{\prime}=C_{e}^{\prime} \times C_{f}^{\prime \prime}=C_{g}^{\prime}
$$

while $C_{e} \times C_{f}^{\prime \prime}=C_{e}^{\prime \prime} \times C_{f}^{\prime \prime}=C_{g}^{\prime \prime}$.

From $C_{0} \times C_{f}=C_{g}$, we obtain as a consequence of the sum theorem 1.61

$$
\left(\underset{0<e^{\prime}<e}{\cup} C_{e^{\prime}}\right) \times C_{f}=\underset{0<\theta^{\prime}<0}{\bigcup} C_{\sigma^{\prime}} \text { or } C_{e}^{\prime} \times C_{f}=C_{o}^{\prime},
$$

since $g=e f /(e+f)$ is continuous and monotonic in $e$ for fixed $f$. By repeating the operation we get since $U_{g^{\prime}<g} C_{g^{\prime}}^{\prime}=C_{0}^{\prime}: C_{e}^{\prime} \times C_{f}^{\prime}=C_{g}^{\prime}$.

Since $C_{\theta}$ has the property $K$ for all $e>0$, as well as the property $\Delta_{0}^{\prime \prime}$, we can also apply the product theorems 1.62 and 1.64 to $C_{e} \times C_{f}=C_{g}$ and obtain $C_{\varepsilon} \times C_{f}^{\prime \prime}=C_{g}^{\prime \prime}$, it being assumed that $f^{\prime}$ runs over a monotonic decreasing sequence approaching $f$, and the corresponding $g^{\prime}$ obviously approaches $g$ in the same way. If we apply the sum theorem 1.61 to this result we obtain by $1.31 \mathrm{c}$

$$
C_{e}^{\prime} \times C_{f}^{\prime \prime}=\bigcup_{h<0} C_{h}^{\prime \prime}=C_{g}^{\prime} \text {. }
$$

Finally to prove that $C_{o}^{\prime \prime} \times C_{f}^{\prime \prime}=C_{o}^{\prime \prime}$, we note that by 1.62 we have

$$
\bigcap_{0^{\prime}>0} C_{0}^{\prime} \times C_{f}^{\prime \prime} \subset \bigcap_{0^{\prime}>0} C_{\sigma^{\prime}}^{\prime \prime}, \text { that is, } C_{0}^{\prime \prime} \times C_{f}^{\prime \prime} \subset C_{0}^{\prime \prime} \text {. }
$$

On the other hand since $C_{e}^{\prime \prime} \supset C_{e}$, we have

$$
C_{g}^{\prime \prime}=C_{0} \times C_{f}^{\prime \prime} \subset C_{\bullet}^{\prime \prime} \times C_{f}^{\prime \prime} \text {, }
$$

from which two statements the desired conclusion follows.

We might note that if in the proof of $C_{\theta}^{\prime} \times C_{f}^{\prime \prime}=C_{o}^{\prime}$ we had started with the identity $C_{e}^{\prime} \times C_{f}=C_{g}^{\prime}$ and been able to apply the product theorem then the equality $C_{b}^{\prime} \times C_{f}^{\prime \prime}=C_{0}^{\prime \prime}$ would have resulted, in other words the product theorem 1.64 is not applicable. Since the 
class $C_{f}$ has the property $\Delta_{0}^{\prime \prime}$, it follows from this that the class $C_{0}^{\prime}$ does not have the property $K$.

For the case where $e=\infty$, we need consider only $C_{\infty}^{\prime}$. We obtain at once for $f>0: C_{\infty}^{\prime} \times C_{f}=C_{\infty}^{\prime}$. From this follows at once: $C_{\infty}^{\prime} \times C_{f}^{\prime \prime}$ $=C_{\infty}^{\prime} \times C_{f}=C_{\infty}^{\prime} \times C_{f}^{\prime}=C_{\infty}^{\prime} \times C_{\infty}^{\prime}=C_{\infty}^{\prime}$.

For the case where $e=0$, we need consider only the class $C_{0}^{\prime \prime}$. Obviously Theorem 1.64 also applies here if $f \neq 0$, that is, we have

$$
C_{0}^{\prime \prime} \times C_{f}=C_{0}^{\prime \prime} \quad \text { for all } 0<f<\infty \text {. }
$$

Consequently for the same range of $f$ we have also

$$
C_{0}^{\prime \prime} \times C_{f}^{\prime}=C_{0}^{\prime \prime} \times C_{f}^{\prime \prime}=C_{0}^{\prime \prime} \times C_{\infty}^{\prime}=C_{0}^{\prime \prime} \text {. }
$$

It is further obvious from 1.62 that $C_{0}^{\prime \prime} \times C_{0}^{\prime \prime} \subset C_{0}^{\prime \prime}$. To prove the inclusion in the other direction, we take an arbitrary sequence $\gamma$ of $C_{0}^{\prime \prime}$ and let $\alpha=\gamma^{1 / 2}$ and $\beta=\gamma^{1 / 2}$, and then obviously $\alpha$ and $\beta$ are of $C_{0}^{\prime \prime}$.

We incorporate all of these results in the form of a theorem.

2.24. The classes of System I between $C_{0}^{\prime \prime}$ and $C_{\infty}^{\prime}$ form a closed system under multiplication $(X)$, the various multiplications can be read

\begin{tabular}{|c|c|c|c|c|c|}
\hline & $C_{0}^{\prime \prime}$ & $C_{f}^{\prime}$ & $C_{f}$ & $C_{f}^{\prime \prime}$ & $C_{\infty}^{\prime}$ \\
\hline$C_{0}^{\prime \prime}$ & $C_{0}^{\prime \prime}$ & $C_{0}^{\prime \prime}$ & $C_{0}^{\prime \prime}$ & $C_{0}^{\prime \prime}$ & $C_{0}^{\prime \prime}$ \\
\hline$C_{e}^{\prime}$ & $C_{0}^{\prime \prime}$ & $C_{g}^{\prime}$ & $C_{g}^{\prime}$ & $C_{g}^{\prime}$ & $C_{\infty}^{\prime}$ \\
\hline$C_{0}$ & $C_{0}^{\prime \prime}$ & $C_{0}^{\prime}$ & $C_{g}$ & $C_{g}^{\prime \prime}$ & $C_{\infty}^{\prime}$ \\
\hline$C_{.}^{\prime \prime}$ & $C_{0}^{\prime \prime}$ & $C_{0}^{\prime}$ & $C_{g}^{\prime \prime}$ & $C_{o}^{\prime \prime}$ & $C_{\infty}^{\prime}$ \\
\hline$C_{\infty}^{\prime}$ & $C_{0}^{\prime \prime}$ & $C_{\infty}^{\prime}$ & $C_{\infty}^{\prime}$ & $C_{\infty}^{\prime}$ & $C_{\infty}^{\prime}$ \\
\hline
\end{tabular}
from the following table, in which $g=e f /(e+f)$ :

We turn next to the extension of 2.21 (b) to the classes $C^{\prime}, C^{\prime \prime}, D^{\prime}$ and $D^{\prime \prime}$. We have:

2.25. THEOREM IV. If $g=e f /(e-f)$ and $e>f>0$, then

$$
C^{\prime} \times D_{-f}=C_{e} \times D_{-f}^{\prime}=C_{e}^{\prime} \times D_{-f}^{\prime}=C_{e}^{\prime} \times D_{-f}^{\prime \prime}=C_{e}^{\prime \prime} \times D_{-f}^{\prime}=D_{-o}^{\prime}
$$

and $C_{e} \times D_{-f}^{\prime \prime}=C_{e}^{\prime \prime} \times D_{-f}=C_{e}^{\prime \prime} \times D_{-f}^{\prime \prime}=D_{-g}^{\prime \prime}$.

Starting from the equation $C_{e} \times D_{-f}=D_{-\sigma}$, the methods of proof used for 2.23 or Theorem III apply here also, with the exception of the characterization of the classes $C_{\theta}^{\prime \prime} \times D_{-f}^{\prime}$ and $C_{\theta}^{\prime \prime} \times D_{-f}$. For the 
class $C_{e}^{\prime \prime} \times D_{-f}^{\prime}$, we note that the product theorem 1.62 applied to $C_{\varepsilon} \times D_{-f} \subset D_{-\sigma}$ yields $C_{e}^{\prime \prime} \times D_{-f} \subset D_{-g}^{\prime \prime}$. Applying the sum theorem 1.61 and also $1.31 \mathrm{c}$ gives $C_{s}^{\prime \prime} \times D_{-f}^{\prime} \subset D_{-g}^{\prime}$. On the other hand $C_{e}^{\prime \prime} \times D_{-f}^{\prime} \supset C_{e} \times D_{-f}^{\prime}=D_{-g}^{\prime}$. So that $C_{e}^{\prime \prime} \times D_{-f}^{\prime}=D_{-g}^{\prime}$.

For the class $C_{e}^{\prime \prime} \times D_{-f}$, we need only show that it contains $D_{-\vartheta}^{\prime \prime}$, as the inverse inclusion is contained in the preceding paragraph. It is not permissible here to use Theorem 1.64 , because $D_{-f}$ does not have the dominance property $K$. Instead we proceed directly. Suppose that $\gamma$ is of $D_{-g}^{\prime \prime}$, that is, there exists a monotonic increasing sequence $g_{n}$ approaching $g$, such that $\gamma$ is of $D_{-\sigma_{n}}$ for every $n$. Let $e_{n}$ be determined from the equation $g_{n}=e_{n} f /\left(e_{n}-f\right)$, so that $e_{n}$ approaches $e$ monotonically decreasing. Since $D_{-\sigma_{n}}=C_{\boldsymbol{e}_{n}} \times D_{-f}$ there exist $\alpha_{n}=\left\{a_{n m}\right\}$ of $C_{\boldsymbol{b}_{n}}$ and $\beta_{n}=\left\{b_{n m}\right\}$ of $D_{-f}$ so that $\gamma=\alpha_{n} \times \beta_{n}$ for every $n$. Let the sequence $d_{n}$, with $0<d_{n}<1$, be such that $\sum d_{n}$ converges. Determine the sequence $m_{n}>m_{n-1}$ so that

$$
\sum_{m=m_{n}}^{\infty} a_{n m}^{e_{n}}<d_{n}, \quad \sum_{m_{n} \leqq m<m_{n+1}} b_{n m}^{-f}>1 .
$$

Define $a_{m}=a_{n m}$ and $b_{m}=b_{n m}$ for $m_{n} \leqq m<m_{n+1}$, with $a_{m}=a_{1 m}$ and $b_{m}=b_{1 m}$ for $m \leqq m_{1}$. Then obviously $\gamma=\alpha \times \beta$. $\alpha$ belongs to $C_{\theta}$ for every $e^{\prime}>e$ since $a_{m}<1$ for $m \geqq m_{1}$, so that if $e^{\prime}>e_{n}, m>m_{1}$, then $a_{m}^{e^{\prime}}<a_{m}^{e_{n}}$ and consequently $\sum_{m_{n}}^{\infty} a_{m}^{e^{\prime}}<\sum d_{n}$. Obviously $\beta$ belongs to $D_{-f}$.

The equation $C_{\theta}^{\prime \prime} \times D_{-f}^{\prime}=D_{-a}^{\prime}$ could also have been deduced by the sum theorem 1.61 from $C_{e}^{\prime \prime} \times D_{-f}=D_{-g}^{\prime \prime}$. Also the case $e=\infty$ which concerns $C_{\infty}^{\prime}$ follows immediately so that we have for $f>0$

$$
C_{\infty}^{\prime} \times D_{-f}=C_{\infty}^{\prime} \times D_{-f}^{\prime}=C_{\infty}^{\prime} \times D_{-f}^{\prime \prime}=D_{-f}^{\prime}
$$

and $C_{\infty}^{\prime} \times D_{-0}^{\prime}=D_{-0}^{\prime}$.

2.26. Theorem V. If $0<e<f$, then $C_{e}^{(1)} \times D_{-f}^{(2)}=-F_{-1}$, where $C_{e}^{(1)}$ may be either $C_{e}^{\prime}, C_{0}$ or $C_{e}^{\prime \prime}$ and $D_{-f}^{(2)}$ may be either $D_{-f}^{\prime}, D_{-f}$ or $D_{-f}^{\prime \prime}$.

This is an immediate consequence of the inclusion theorem 1.65. For the case $e=f$, Theorem IV separates into two parts, namely

$$
\begin{aligned}
C_{\odot} \times D_{-\theta} & =C_{\theta}^{\prime} \times D_{-\theta}=C_{\theta} \times D_{-\theta}^{\prime}=C_{e}^{\prime} \times D_{-\theta}^{\prime} \\
& =C_{e}^{\prime} \times D_{-e}^{\prime \prime}=C_{e}^{\prime \prime} \times D_{-e}^{\prime}=-F_{-1} .
\end{aligned}
$$

This includes also the case where $e=0$ or $\infty$, namely

$$
C_{\infty}^{\prime} \times D_{-\infty}^{\prime \prime}=C_{0}^{\prime \prime} \times D_{-0}^{\prime}=-F_{-1} .
$$

On the other hand 


$$
C_{\bullet}^{\prime \prime} \times D_{-\bullet}=C_{e} \times D_{-{ }_{0}}^{\prime \prime}=C_{\bullet}^{\prime \prime} \times D_{-\odot}^{\prime \prime}=D_{-\infty}^{\prime \prime}
$$

For 2.27 the first class is covered in 2.21(c), the next three classes each are contained in $C_{8} \times D_{-}$and by 2.22 contain $-F_{-1}$. For the last two the method of procedure is

$$
\begin{aligned}
& C_{\bullet}^{\prime} \times D_{-\bullet}^{\prime \prime}=\left(\bigcup_{f<e} C_{f}\right) \times D_{-\theta}^{\prime \prime}=\bigcup_{f<e}\left(C_{f} \times D_{-e}^{\prime \prime}\right)=-F_{-1}, \\
& C_{e}^{\prime \prime} \times D_{-\bullet}^{\prime}=C_{e}^{\prime \prime} \times\left(\bigcup_{f>e} D_{-f}\right)=\bigcup_{f>e}\left(C_{-\bullet}^{\prime \prime} \times D_{-f}\right)=-F_{-1} .
\end{aligned}
$$

The first of these two is also valid for $e=\infty$ and the second for $e=0$, giving 2.28.

For 2.29 the methods used in the proof of the corresponding results in 2.25 are applicable.

To finish this group of cases, we need to consider yet the possibility of having $e=0$, that is, the class $C_{0}^{\prime \prime} ; f=0$, that is, the class $D_{-0}^{\prime}$; and the case $f=\infty$, that is, $D_{-\infty}^{\prime \prime}$.

For $C_{0}^{\prime \prime}$ we have:

2.31. $C_{0}^{\prime \prime} \times P=-F_{-1}$ for every class $P$ between and including $-F_{-1}$ and $D_{-0}^{\prime}$.

In 2.28 it was shown that $C_{0}^{\prime \prime} \times D_{-0}^{\prime}=-F_{-1}$. So it remains to prove that $C_{0}^{\prime \prime} \times-F_{-1}=-F_{-1}$. It is obvious that since $C_{0}^{\prime \prime} \subset F$, we have $C_{0}^{\prime \prime} \times-F_{-1} \subset-F_{-1}$. On the other hand suppose $\gamma=\left\{c_{n}\right\}$ is of $-F_{-1}$ and let $\alpha_{0}=\left\{a_{0 n}\right\}$ be any element of $C_{0}^{\prime \prime}$, that is, $\sum_{n} a_{0 n}^{e}<\infty$ for $e>0$. Select a sequence $n_{k}$ so that $c_{n_{k}}<a_{0 k} / k$. Then define $a_{n}=a_{0 n}$ for $n \neq n_{k}$ and $a_{n}=a_{0 k}$ for $n=n_{k}$. Then obviously $\sum_{n} a_{n}^{e}<2 \sum_{n} a_{0 n}^{e}$, for $e>0$, that is, $\alpha$ is of $C_{0}^{\prime \prime}$. On the other hand since $b_{n_{k}}=c_{n_{k}} / a_{n_{k}}=c_{n_{k}} / a_{0 k}<1 / k$, $\beta$ is of $-F_{-1}$.

For $D_{-0}^{\prime}$ we have:

2.32. $D_{-0}^{\prime} \times P=D_{-0}^{\prime}$ for all classes $P$ between $C_{0}^{\prime \prime}$ and $F$, including $F$.

Obviously $D_{-0}^{\prime} \times F=D_{-0}^{\prime}$. It is then sufficient to prove that $D_{-0}^{\prime} \times C_{\theta}=D_{-0}^{\prime}$ for all $e>0$, which follows immediately from 2.25, namely, $C_{\varepsilon} \times D_{-f}=D_{-g}$, by applying the sum theorem 1.61 .

For $D_{-\infty}^{\prime \prime}$ we have:

2.33. $D_{-\infty}^{\prime \prime} \times P=-F_{-1}$ for all classes $P$ between and including $C_{0}^{\prime \prime}$ and $C_{\infty}^{\prime}$.

The equality $D_{-\infty}^{\prime \prime} \times C_{0}^{\prime \prime}=-F_{-1}$ is included in 2.31. Since for every $e>0, C_{e}$ has the dominance property $K$, we can apply the product theorem 1.64 to $C_{\theta} \times D_{-f}=-F_{-1}$ to obtain $C_{e} \times D_{-\infty}^{\prime \prime}=-F_{-1}$, for all $e>0$. Applying the sum theorem 1.61 to this equality yields $C_{\infty}^{\prime} \times D_{-\infty}^{\prime \prime}$ $=-F_{-1}$. 
2.4. The classes between $L_{0}$ and $-L_{\infty}$. We consider next results involving the classes $L_{0}, F \cap-F_{-1}, F,-F_{-1}, F \cup-F_{-1},-L_{\infty}$, so far as they have not been previously treated. We have:

2.41. $F \times P=P$ for every class $P$ of System $\mathrm{I}$.

Since the sequence $\alpha=\{1\}$ is of $F$, it is obvious that $F \times P \supset P$. On the other hand, since all classes $P$ of System I have the property $\Delta_{0}^{\prime \prime}$, $F \times P \subset P$.

2.42. $-F_{-1} \times P=-F_{-1}$ for any class $P$ contained in or equal to $F$.

Since $-F_{-1} \times F \subset-F_{-1}$, it follows that $-F_{-1} \times P C-F_{-1}$ for all $P$ contained in $F$. On the other hand suppose $\gamma=\left\{c_{n}\right\}$ is of $-F_{-1}$. Select a $\beta_{0}=\left\{b_{0 n}\right\}$ of $P$, and the sequence $n_{k}$ so that $c_{n_{k}}<b_{0 k} / k$. Let $b_{n}=b_{0 n}$ if $n \neq n_{k}$, and $b_{n}=b_{0 k}$ if $n=n_{k}$. Then if $a_{n}=c_{n} / b_{n}$, it follows that $\lim \inf _{n} a_{n}=0$, that is, $\alpha$ is of $-F_{-1}$. But for the classes $P$ of System I, $\beta=\left\{b_{n}\right\}$ is of $P$ if $\beta_{0}$ is.

Since $-F_{-1} \subset F \cup-F_{-1} \subset-L_{\infty} \subset D_{-\infty}^{\prime \prime}$, we can conclude from 2.33 and 2.42 that:

2.43. $-F_{-1} \times P=\left(F \cup-F_{-1}\right) \times P=\left(-L_{\infty}\right) \times P=D_{-\infty}^{\prime \prime} \times P=-F_{-1}$ for all classes $P$ between and including $C_{0}^{\prime \prime}$ and $C_{\infty}^{\prime}$.

2.44. $L_{0} \times P=P$ for all classes $P$ between and including $C_{0}^{\prime \prime}$ and $L_{0}$, and also between and including $D_{-\infty}^{\prime \prime}$ and $D_{-0}^{\prime}$.

Since $L_{0} \subset F$, and $F \times P=P$ for all $P$ it follows that $L_{0} \times P \subset P$ for all $P$. An analysis of the statement $L_{0} \times P \supset P$ shows that it is equivalent to the fact that the classes $P$ have the dominance property ${ }^{7} K_{0}$, namely, that if $\alpha=\left\{a_{n}\right\}$ is of $P$, then there exists in $P$ an $\alpha_{0}=\left\{a_{0 n}\right\}$ such that $\lim _{n} a_{n} / a_{0 n}=0$, which in turn is related to the "no last absolutely convergent or divergent series" theorem. The proof for $C_{1}=C$ and consequently for $C_{\theta}$ can be based on the Abel-Dini Theorem for convergent series. ${ }^{8}$ Let $\gamma=\left\{c_{n}\right\}$ be of $C$ and let $\rho=\left\{r_{n}\right\}=\sum_{n}^{\infty} c_{m}$. Then $\gamma \times \rho^{-1+e}$ is of $C$ for $e>0$. If we set $\alpha=\gamma \times \rho^{-1+e}$, then $\beta=\rho^{1-e}$ which is of $L_{0}$. For the other classes between and including $C_{0}^{\prime \prime}$ and $C_{\infty}^{\prime}$, we can apply 1.61 and 1.64 , since $L_{0}$ has the property $K$. For $L_{0} \times L_{0} \subset L_{0}$, we take $\alpha=\beta=\gamma^{1 / 2}$. For the class $D_{-1}$, we utilize in a similar way the Abel-Dini Theorem for divergent series, that is, define $\alpha=\gamma \times \sigma$, where $\sigma=\left\{\left(\sum_{1}^{n} c_{m}\right)^{-1}\right\}$ so that $\beta=\sigma^{-1}$ which is of $L_{0}$. The extension to $D_{-\varepsilon}$ and the other classes between $D_{-\infty}^{\prime \prime}$ and $D_{-0}^{\prime}$ is then similar to that for the $C$.

2.45. $\left(F \cap-F_{-1}\right) \times P=P$ for all $P$ between $C_{0}^{\prime \prime}$ and $L_{0}$ inclusive and $D_{-\infty}^{\prime \prime}$ and $D_{-0}^{\prime}$ inclusive.

This is a corollary to 2.44 and 2.41 in view of the fact that $F \supset F \cap-F_{-1} \supset L_{0}$.

${ }^{7}$ See Moore, loc. cit. p. 44.

${ }^{8}$ See Knopp, loc. cit. p. 293. 
These theorems while they cover a wide range still leave a few particular cases. We have:

2.46. $-L_{\infty} \times L_{\theta}=-F_{-1} ;-L_{\infty} \times\left(F \cap-F_{-1}\right)=-L_{\infty}$.

From the definitions it is obvious that $-L_{\infty} \times L_{0} \subset-F_{-1}$, for if $\alpha=\left\{a_{n}\right\}$ is of $-L_{\infty}$, then $a_{n}$ contains a bounded subsequence, so that if $\beta=\left\{b_{n}\right\}$ is of $L_{0}$, then $\lim \inf _{n} a_{n} b_{n}=0$. On the other hand suppose that $\gamma=\left\{c_{n}\right\}$ is of $-F_{-1}$. Let $\alpha_{0}=\left\{a_{0_{n}}\right\}$ be any element in $L_{0}$ and select an increasing sequence $n_{k}$ so that $c_{n_{k}} \leqq a_{0 k}$. Then define $\alpha=\left\{a_{n}\right\}$ so that $a_{n}=a_{0 n}$ for $n \neq n_{k}$ and $a_{n}=a_{0 k}$ for $n=n_{k}$. Then $b_{n_{k}}=c_{n_{k}} / a_{n_{k}} \leqq 1$ so that $\beta$ is of $-L_{\infty}$ and $\alpha$ is of $L_{0}$.

For the second part, it is again obvious that $-L_{\infty} \times\left(F \cap-F^{-1}\right)$ $C-L_{\infty}$. On the other hand let $\gamma=\left\{c_{n}\right\}$ be of $-L_{\infty}$, and let $\left\{c_{n_{k}}\right\}$ be a bounded subsequence of $\left\{c_{n}\right\}$. Let $a_{n}=c_{n}$ if $n=n_{k}$ and $a_{n}=c_{n} n$ if $n \neq n_{k}$. Then $b_{n}=1$ for $n=n_{k}$ but $b_{n}=1 / n$ for $n \neq n_{k}$ so that $\alpha$ is of $-L_{\infty}$ and $\beta$ is of $F \cap-F_{-1}$.

2.47. $L_{0} \times\left(F \cup-F_{-1}\right)=-F_{-1}$.

This is a consequence of 2.42 , namely, $L_{0} \times\left(-F_{-1}\right)=-F_{-1}$, and 2.46, namely, $L_{0} \times\left(-L_{\infty}\right)=-F_{-1}$, and the fact that $-F_{-1} \subset F \cup-F_{-1}$ $\subset-L_{\infty}$.

2.48. $\left(F \cup-F_{-1}\right) \times\left(F \cap-F_{-1}\right)=-F_{-1}$.

We have $\left(F \cap-F_{-1}\right) \times F=F \cap-F_{-1}$ and $\left(F \cap-F_{-1}\right) \times-F_{-1}$ $=-F_{-1}$, by 2.41 and 2.42 . By addition we then get 2.48 .

2.49. $\left(F \cap-F_{-1}\right) \times\left(F \cap-F_{-1}\right)=F \cap-F_{-1}$.

This is obvious.

2.5. The classes $V$ and 0 . We finally consider the classes $V$ and 0 and note:

2.51. $P \times V=V$ for all $P$ of System I excepting 0 .

2.52. $P \times 0=0$ for all $P$ of System $\mathrm{I}$.

These are self-evident.

It may however be of interest to apply Theorems 1.52 to these results in order to obtain corresponding division theorems. Thus

$$
\begin{aligned}
& 0 / 0=-\left(0 \times-0_{-1}\right)_{-1}=-(0 \times V)_{-1}=V, \\
& V / V=-\left(V \times V_{-1}\right)_{-1}=-(V \times 0)_{-1}=V, \\
& V / 0=-\left(0 \times-V_{-1}\right)_{-1}=(0 \times 0)_{-1}=V, \\
& 0 / V=-\left(V \times-0_{-1}\right)_{-1}=-(V \times V)_{-1}=0 .
\end{aligned}
$$

Of these $V / 0=V$ is an example available for 1.510 and 1.512.

We have collected all of the results of this part in the form of a table, indicating in each place the particular theorem on which the entry is based. Because of the fact that $P \times Q=Q \times P$, it is obvious that it is sufficient to fill in only one half of the entries, as the re- 


\begin{tabular}{|c|c|c|c|c|c|c|c|c|c|c|c|c|}
\hline & 0 & $C^{\prime \prime}{ }_{0}$ & $C^{\prime}$ & $C_{0}$ & $C^{\prime \prime}$. & $C_{f}^{\prime}$ & $C_{f}$ & $C^{\prime \prime}$ & $C_{\infty}^{\prime}$ & $L_{0}$ & $F \cap-F_{-1}$ & $F$ \\
\hline 0 & $\begin{array}{c}0 \\
2.52 \\
\end{array}$ & $\begin{array}{c}0 \\
2.52 \\
\end{array}$ & $\begin{array}{c}0 \\
2.52 \\
\end{array}$ & $\begin{array}{c}0 \\
2.52 \\
\end{array}$ & $\begin{array}{c}0 \\
2.52 \\
\end{array}$ & $\begin{array}{c}0 \\
2.52 \\
\end{array}$ & $\begin{array}{c}0 \\
2.52 \\
\end{array}$ & $\begin{array}{c}0 \\
2.52 \\
\end{array}$ & $\begin{array}{c}0 \\
2.52 \\
\end{array}$ & $\begin{array}{c}0 \\
2.52 \\
\end{array}$ & $\begin{array}{c}0 \\
2.52\end{array}$ & $\begin{array}{c}0 \\
2.52\end{array}$ \\
\hline$C^{\prime \prime}{ }_{0}$ & & $\begin{array}{l}C^{\prime \prime}{ }_{0} \\
2.24\end{array}$ & $\begin{array}{l}C^{\prime \prime} 0 \\
2.24\end{array}$ & $\begin{array}{l}C^{\prime \prime}{ }_{0} \\
2.24 \\
\end{array}$ & $\begin{array}{l}C^{\prime \prime}{ }_{0} \\
2.24\end{array}$ & $\begin{array}{l}C^{\prime \prime}{ }_{0} \\
2.24\end{array}$ & $\begin{array}{l}C^{\prime \prime} 0 \\
2.24\end{array}$ & $\begin{array}{l}C^{\prime \prime}{ }_{0} \\
2.24\end{array}$ & $\begin{array}{l}C^{\prime \prime}{ }_{0} \\
2.24\end{array}$ & $\begin{array}{l}C^{\prime \prime} 0 \\
2.44 \\
\end{array}$ & $\begin{array}{r}C^{\prime \prime} 0 \\
2.45 \\
\end{array}$ & $\begin{array}{r}C^{\prime \prime}{ }_{0} \\
2.41 \\
\end{array}$ \\
\hline$C^{\prime}$ & & & $\begin{array}{l}C^{\prime}{ }_{e / 2} \\
2.23 \\
\end{array}$ & $\begin{array}{l}C^{\prime} / 2 \\
2.23\end{array}$ & $\begin{array}{l}C^{\prime} / 2 \\
2.23\end{array}$ & $\begin{array}{l}C^{\prime}{ }_{g} \\
2.23\end{array}$ & $\begin{array}{l}C^{\prime}{ }_{0} \\
2.23\end{array}$ & $\begin{array}{l}C^{\prime}{ }^{\prime} \\
2.23 \\
\end{array}$ & $\begin{array}{l}C^{\prime} \infty \\
2.24\end{array}$ & $\begin{array}{l}C^{\prime} \\
2.44\end{array}$ & $\begin{array}{l}C^{\prime}{ }^{\prime} \\
2.45\end{array}$ & $\begin{array}{l}C^{\prime} \\
2.41\end{array}$ \\
\hline$C_{6}$ & & & & $\begin{array}{l}C_{\ell / 2} \\
2.21 \\
\end{array}$ & $\begin{array}{l}C^{\prime \prime}{ }_{e / 2} \\
2.23\end{array}$ & $\begin{array}{l}C^{\prime}{ }_{0} \\
2.23 \\
\end{array}$ & $\begin{array}{l}C_{g} \\
2.21 \\
\end{array}$ & $\begin{array}{l}C^{\prime \prime}{ }^{\prime} \\
2.23 \\
\end{array}$ & $\begin{array}{l}C_{\infty}^{\prime} \\
2.24\end{array}$ & \begin{tabular}{|l|}
$C_{6}$ \\
2.44 \\
\end{tabular} & $\begin{array}{l}C_{6} \\
2.45\end{array}$ & $\begin{array}{c}C_{e} \\
2.41\end{array}$ \\
\hline$C^{\prime \prime}$ & & & & & $\begin{array}{l}C^{\prime \prime}{ }_{e / 2} \\
2.23 \\
\end{array}$ & $\begin{array}{l}C^{\prime} g \\
2.23 \\
\end{array}$ & $\begin{array}{c}C^{\prime \prime}{ }_{0} \\
2.23 \\
\end{array}$ & $\begin{array}{r}C^{\prime \prime}{ }_{g} \\
2.23 \\
\end{array}$ & $\begin{array}{r}C^{\prime} \\
2.24 \\
\end{array}$ & $\begin{array}{r}C^{\prime \prime}{ }_{0} \\
2.44 \\
\end{array}$ & $\begin{array}{l}C^{\prime \prime} \\
2 . \dot{4} 5 \\
\end{array}$ & $\begin{array}{r}C^{\prime \prime} \\
2.41 \\
\end{array}$ \\
\hline$C_{f}^{\prime}$ & & & & & & $\begin{array}{l}C_{f / 2}^{\prime} \\
2.23 \\
\end{array}$ & $\begin{array}{l}C_{f / 2}^{\prime} \\
2.23 \\
\end{array}$ & $\begin{array}{l}C_{f / 2}^{\prime} \\
2.23 \\
\end{array}$ & $\begin{array}{l}C_{\infty}^{\prime} \\
2.24\end{array}$ & $\begin{array}{l}C_{f}^{\prime} \\
2.44 \\
\end{array}$ & $\begin{array}{c}C_{f}^{\prime} \\
2.45 \\
\end{array}$ & $\begin{array}{l}C_{\prime}^{\prime}{ }_{f} \\
2.41\end{array}$ \\
\hline$C_{f}$ & & & & & & & $\begin{array}{l}C_{f / 2} \\
2.21 \\
\end{array}$ & $\begin{array}{l}C^{\prime \prime} f / 2 \\
2.23 \\
\end{array}$ & $\begin{array}{l}C^{\prime}{ }_{\infty} \\
2.24\end{array}$ & $\begin{array}{l}C_{f} \\
2.44 \\
\end{array}$ & $\begin{array}{l}C_{f} \\
2.45\end{array}$ & $\begin{array}{c}C_{f} \\
2.41 \\
\end{array}$ \\
\hline$C_{f}^{\prime \prime}$ & & & & & & & & $\begin{array}{l}C^{\prime \prime} f / 2 \\
2.23 \\
\end{array}$ & $\begin{array}{l}C_{\infty}^{\prime} \\
2.24 \\
\end{array}$ & $\begin{array}{l}C^{\prime \prime} f_{f} \\
2.44 \\
\end{array}$ & $\begin{array}{l}C^{\prime \prime}{ }_{f} \\
2.45\end{array}$ & $\begin{array}{l}C^{\prime \prime}{ }_{f} \\
2.41 \\
\end{array}$ \\
\hline$C_{\infty}^{\prime}$ & & & & & & & & & \begin{tabular}{|l|}
$C^{\prime} \infty$ \\
2.24 \\
\end{tabular} & $\begin{array}{l}C^{\prime} \infty \\
2.44 \\
\end{array}$ & $\begin{array}{l}C^{\prime}{ }_{\infty} \\
2.45 \\
\end{array}$ & $\begin{array}{r}C^{\prime}{ }_{\infty} \\
2.41 \\
\end{array}$ \\
\hline$L_{0}$ & & & & & & & & & & $\begin{array}{l}L_{0} \\
2.44 \\
\end{array}$ & $\begin{array}{c}L_{0} \\
2.45 \\
\end{array}$ & $\begin{array}{c}L_{0} \\
2.41 \\
\end{array}$ \\
\hline$\overline{F \cap-F_{-1}}$ & & & & & & & & & & & $\begin{array}{c}F \cap-F-1 \\
2.49\end{array}$ & $\begin{array}{l}F \cap-F_{-1} \\
2.41 \\
\end{array}$ \\
\hline$F$ & & & & & & & & & & & & $\begin{array}{l}F \\
2.41 \\
\end{array}$ \\
\hline$-F_{-1}$ & & & & & & & & & & & & \\
\hline$F \cup-F_{-1}$ & & & & & & & & & & & & \\
\hline$-L_{\infty}$ & & & & & & & & & & & & \\
\hline$D^{\prime \prime}-\infty$ & & & & & & & & & & & & \\
\hline$D_{-f}^{\prime}$ & & & & & & & & & & & & \\
\hline$D_{-f}$ & & & & & & & & & & & & \\
\hline$D^{\prime \prime}-f$ & & & & & & & & & & & & \\
\hline$D^{\prime} \rightarrow$ & & & & & & & & & & & & \\
\hline$D_{-}$ & & & & \multicolumn{7}{|c|}{$0<e<f . \quad g=e f /(e+f) . \quad g^{\prime}=e f /(f-e)$} & & \\
\hline$D^{\prime \prime}$ & & & & & & & & & & & & \\
\hline$D_{-0}^{\prime}$ & & & & & & & & & & & & \\
\hline$V$ & & & & & & & & & & & & \\
\hline
\end{tabular}




\begin{tabular}{|c|c|c|c|c|c|c|c|c|c|c|c|}
\hline$-F_{-1}$ & $\left|F \cup-F_{-}\right|$ & $-L_{\infty}$ & $D^{\prime \prime}-\infty$ & $D_{-f}^{\prime}$ & $D_{-f}$ & $D^{\prime \prime}-f$ & $D_{\rightarrow}^{\prime}$ & $D_{\rightarrow}$ & $D^{\prime \prime} \rightarrow$ & $D_{-0}^{\prime}$ & $V$ \\
\hline $\begin{array}{c}0 \\
2.52\end{array}$ & $\begin{array}{c}0 \\
2.52\end{array}$ & $\begin{array}{c}0 \\
2.52\end{array}$ & $\begin{array}{c}0 \\
2.52\end{array}$ & \begin{tabular}{c|}
0 \\
2.52 \\
\end{tabular} & \begin{tabular}{c|}
0 \\
2.52
\end{tabular} & $\begin{array}{c}0 \\
2.52\end{array}$ & $\begin{array}{c}0 \\
2.52\end{array}$ & $\begin{array}{c}0 \\
2.52\end{array}$ & $\begin{array}{c}0 \\
2.52\end{array}$ & $\begin{array}{c}0 \\
2.52\end{array}$ & $\begin{array}{c}0 \\
2.52\end{array}$ \\
\hline$\frac{-F_{-1}}{2.31}$ & $\frac{-F_{-1}}{2.31}$ & $\begin{array}{l}-F_{-1} \\
2.31\end{array}$ & $\frac{-F_{-1}}{2.31}$ & $\mid \begin{array}{l}-F_{-1} \\
2.31\end{array}$ & $\frac{-F_{-1}}{2.31}$ & $\frac{-F_{-1}}{2.31}$ & $\frac{-F_{-1}}{2.31}$ & $\frac{-F_{-1}}{2.31}$ & $\frac{-F_{-1}}{2.31}$ & $\mid \begin{array}{l}-F_{-1} \\
2.28\end{array}$ & $\begin{array}{l}V \\
2.51\end{array}$ \\
\hline$\overline{2 . F_{-1}}$ & $\frac{-F_{-1}}{2.43}$ & $\overline{-F_{-1}}$ & $\overline{-F_{-1}}$ & $\begin{array}{c}-F_{-1} \\
2.26\end{array}$ & $\begin{array}{l}-F_{-1} \\
2.26\end{array}$ & $\overline{-F_{-1}}$ & $\overline{-F_{-1}}$ & $\overline{-F_{-1}}$ & $\overline{-F_{-1}}$ & $\begin{array}{l}D_{-00}^{\prime} \\
2.32\end{array}$ & $\begin{array}{c}V \\
2.51\end{array}$ \\
\hline $\begin{array}{l}-F_{-1} \\
2.42\end{array}$ & $\begin{array}{l}-F_{-1} \\
2.43\end{array}$ & $\begin{array}{l}-F_{-1} \\
2.43\end{array}$ & $-F_{-1}$ & $-F_{-1}$ & $\begin{array}{l}-F_{-1} \\
2.26\end{array}$ & $\begin{array}{l}-F_{-1} \\
2.26\end{array}$ & $-F_{\overline{1}^{1}}$ & $\begin{array}{l}-F_{-1} \\
2.27\end{array}$ & $\begin{array}{l}D^{\prime \prime}-\infty \\
2.29\end{array}$ & $\begin{array}{l}D^{\prime}{ }_{-0} \\
2.32\end{array}$ & $\begin{array}{c}V \\
2.51\end{array}$ \\
\hline $\begin{array}{l}-F_{-1} \\
2.42\end{array}$ & $\overline{2}^{-F_{-1}}$ & $\overline{2 . F_{-1}}$ & $\frac{-F_{-1}}{2.33}$ & $\frac{-F_{-1}}{2.26}$ & $\overline{-F_{-1}}$ & $\overline{-F_{-1}}$ & $\overline{-F_{-1}}$ & $\begin{array}{l}D^{\prime \prime}-\infty \\
2.29\end{array}$ & $\begin{array}{l}D^{\prime \prime}-\infty \\
2.27\end{array}$ & $\begin{array}{l}D^{\prime}{ }^{-0} \\
2.32\end{array}$ & $\begin{array}{c}V \\
2.51\end{array}$ \\
\hline$\frac{-F_{-1}}{2.42}$ & $\frac{-F_{-1}}{2.43}$ & $\frac{-F_{-1}}{2.43}$ & $\frac{-F_{-1}}{2.33}$ & $\frac{-F_{-1}}{2.27}$ & $\frac{-F_{-1}}{2.27}$ & $\frac{-F_{-1}}{2.27}$ & $\begin{array}{l}D^{\prime}{ }_{-a^{\prime}} \\
2.25\end{array}$ & $\begin{array}{l}D^{\prime}{ }_{-g^{\prime}} \\
2.25\end{array}$ & $\begin{array}{l}D^{\prime}{ }_{-g^{\prime}} \\
2.25\end{array}$ & $\begin{array}{l}D^{\prime}-0 \\
2.32\end{array}$ & $\begin{array}{c}V \\
2.51\end{array}$ \\
\hline $\begin{array}{l}-F_{-1} \\
2.42\end{array}$ & $\frac{-F_{-1}}{2.43}$ & $\begin{array}{l}-F_{-1} \\
2.43\end{array}$ & $\frac{-F_{-1}}{2.33}$ & $\frac{-F_{-1}}{2.27}$ & $\frac{-F_{-1}}{2.27}$ & $\begin{array}{l}D^{\prime \prime} \\
2.29\end{array}$ & $\begin{array}{l}D^{\prime}-q^{\prime} \\
2.25\end{array}$ & $\begin{array}{l}D_{-g} \\
2.21\end{array}$ & $\begin{array}{l}D^{\prime \prime} \\
2.25\end{array}$ & $\begin{array}{l}D^{\prime}-0, \\
2.32\end{array}$ & $\begin{array}{c}V \\
2.51\end{array}$ \\
\hline $\begin{array}{l}-F_{-1} \\
2.42\end{array}$ & $\begin{array}{l}-F_{-1} \\
2.43\end{array}$ & $\begin{array}{l}-F_{-1} \\
2.43\end{array}$ & $\frac{-F_{-1}}{2.33}$ & $\begin{array}{l}-F_{-1} \\
2.27^{-}\end{array}$ & $\begin{array}{l}D^{\prime \prime} \\
2.29^{-\infty}\end{array}$ & $\begin{array}{l}D^{\prime \prime} \\
2.29^{-\infty}\end{array}$ & $\begin{array}{l}D^{\prime}{ }^{\prime}{ }^{\prime} \\
2.25\end{array}$ & $\begin{array}{l}D^{\prime \prime}-g^{\prime} \\
2.25\end{array}$ & $\begin{array}{l}D^{\prime \prime}-g^{\prime} \\
2.25\end{array}$ & $\begin{array}{l}D^{\prime}-0 \\
2.32\end{array}$ & $\begin{array}{r}V \\
2.51\end{array}$ \\
\hline $\begin{array}{l}-F_{-1} \\
2.42\end{array}$ & $\begin{array}{l}-F_{-1} \\
2.43\end{array}$ & $\begin{array}{l}-F_{-1} \\
2.43\end{array}$ & $\frac{-F_{-1}}{2.33}$ & $\begin{array}{l}D^{\prime}-{ }^{\prime} \\
2.25\end{array}$ & $\begin{array}{l}D^{\prime}-{ }^{\prime} \\
2.25\end{array}$ & $\begin{array}{l}D^{\prime}-f \\
2.25\end{array}$ & $\begin{array}{l}D^{\prime}-{ }^{-6} \\
2.25\end{array}$ & $\begin{array}{l}D^{\prime}-{ }^{e} \\
2.25\end{array}$ & $\begin{array}{l}D^{\prime}-{ }^{6} \\
2.25\end{array}$ & $\begin{array}{l}D^{\prime}-0 \\
2.25\end{array}$ & $\begin{array}{c}V \\
2.51\end{array}$ \\
\hline $\begin{array}{l}-F_{-1} \\
2.42^{2}\end{array}$ & $\overline{2.47}$ & $\begin{array}{l}-F_{-1} \\
2.46\end{array}$ & $\begin{array}{l}D^{\prime \prime}-\infty \\
2.44\end{array}$ & $\begin{array}{l}D^{\prime}-f \\
2.44\end{array}$ & $\begin{array}{l}D_{-f} \\
2.44\end{array}$ & $\begin{array}{l}D^{\prime \prime}-f \\
2.44\end{array}$ & $\begin{array}{l}D^{\prime}-\dot{6} \\
2.44\end{array}$ & $\begin{array}{l}D_{-6} \\
2.44\end{array}$ & $\begin{array}{l}D^{\prime \prime}-{ }^{\circ} \\
2.44\end{array}$ & $\begin{array}{l}D^{\prime}-0 \\
2.44\end{array}$ & $\begin{array}{c}V \\
2.51 \\
\end{array}$ \\
\hline $\begin{array}{l}-F_{-1} \\
2.42\end{array}$ & $\begin{array}{l}-F_{-1} \\
2.48\end{array}$ & $\begin{array}{l}-F_{-1} \\
2.47\end{array}$ & $\begin{array}{l}D^{\prime \prime}-\infty \\
2.45\end{array}$ & $\begin{array}{l}D^{\prime}-f \\
2.45 \\
\end{array}$ & $\begin{array}{l}D_{-f} \\
2.45 \\
\end{array}$ & $\begin{array}{l}D^{\prime \prime}-f \\
2.45\end{array}$ & $\begin{array}{l}D^{\prime}-\overrightarrow{4} 5 \\
2.45\end{array}$ & $\begin{array}{l}D_{-\bullet} \\
2.45 \\
\end{array}$ & $\begin{array}{l}D^{\prime \prime}-8 \\
2.45\end{array}$ & $\begin{array}{l}D^{\prime}-0 \\
2.45 \\
\end{array}$ & $\begin{array}{c}V \\
2.51 \\
\end{array}$ \\
\hline$\overline{2 . F_{-1}}$ & $F \cup \frac{U_{2.41}}{F_{-1}}$ & $\begin{array}{l}-L_{\infty} \\
2.41\end{array}$ & $\begin{array}{l}D^{\prime \prime}-\infty \\
2.41\end{array}$ & $\begin{array}{l}D^{\prime}-f \\
2.41\end{array}$ & $\begin{array}{l}D_{-f} \\
2.41\end{array}$ & $\begin{array}{l}D^{\prime \prime}-f \\
2.41\end{array}$ & $\begin{array}{l}D^{\prime}-a \\
2.41\end{array}$ & $\begin{array}{l}D \\
2.41\end{array}$ & $\begin{array}{l}D^{\prime \prime} \\
2.41\end{array}$ & $\begin{array}{l}D^{\prime}-0 \\
2.41\end{array}$ & $\begin{array}{c}V \\
2.51\end{array}$ \\
\hline \multirow[t]{12}{*}{$\begin{array}{c}V \\
2.12\end{array}$} & $\begin{array}{c}V \\
2.12\end{array}$ & $\begin{array}{c}V \\
2.12\end{array}$ & $\begin{array}{c}V \\
2.12\end{array}$ & $\begin{array}{c}V \\
2.12\end{array}$ & $\begin{array}{c}V \\
2.12\end{array}$ & $\begin{array}{c}V \\
2.12\end{array}$ & $\begin{array}{c}V \\
2.12\end{array}$ & $\begin{array}{c}V \\
2.12\end{array}$ & $\begin{array}{c}V \\
2.12\end{array}$ & $\begin{array}{c}V \\
2.12\end{array}$ & $\begin{array}{c}V \\
2.51\end{array}$ \\
\hline & $\begin{array}{c}V \\
2.12 \\
\end{array}$ & $\begin{array}{c}V \\
2.12 \\
\end{array}$ & $\begin{array}{c}V \\
2.12 \\
\end{array}$ & $\begin{array}{c}V \\
2.12 \\
\end{array}$ & $\begin{array}{c}V \\
2.12 \\
\end{array}$ & $\begin{array}{c}V \\
2.12 \\
\end{array}$ & $\begin{array}{c}V \\
2.12 \\
\end{array}$ & $\begin{array}{c}V \\
2.12 \\
\end{array}$ & $\begin{array}{c}V \\
2.12 \\
\end{array}$ & $\begin{array}{c}V \\
2.12 \\
\end{array}$ & $\begin{array}{c}V \\
2.51 \\
\end{array}$ \\
\hline & & $\begin{array}{c}V \\
2.12\end{array}$ & $\begin{array}{c}V \\
2.12\end{array}$ & $\begin{array}{c}V \\
2.12\end{array}$ & $\begin{array}{c}V \\
2.12\end{array}$ & $\begin{array}{c}V \\
2.12\end{array}$ & $\begin{array}{c}V \\
2.12\end{array}$ & $\begin{array}{c}V \\
2.12\end{array}$ & $\begin{array}{c}V \\
2.12\end{array}$ & $\begin{array}{c}V \\
2.12 \\
\end{array}$ & $\begin{array}{c}V \\
2.51\end{array}$ \\
\hline & & & $\begin{array}{c}V \\
2.12\end{array}$ & $\begin{array}{c}V \\
2.12 \\
\end{array}$ & $\begin{array}{c}V \\
2.12 \\
\end{array}$ & $\begin{array}{c}V \\
2.12 \\
\end{array}$ & $\begin{array}{c}V \\
2.12 \\
\end{array}$ & $\begin{array}{c}V \\
2.12 \\
\end{array}$ & $\begin{array}{r}V \\
2.12 \\
\end{array}$ & $\begin{array}{c}V \\
2.12 \\
\end{array}$ & $\begin{array}{c}V \\
2.51 \\
\end{array}$ \\
\hline & & & & $\begin{array}{c}V \\
2.12\end{array}$ & $\begin{array}{c}V \\
2.12\end{array}$ & $\begin{array}{c}V \\
2.12\end{array}$ & $\begin{array}{c}V \\
2.12\end{array}$ & $\begin{array}{c}V \\
2.12\end{array}$ & $\begin{array}{c}V \\
2.12\end{array}$ & $\begin{array}{c}V \\
2.12\end{array}$ & $\begin{array}{c}V \\
2.51\end{array}$ \\
\hline & & & & & $\begin{array}{c}V \\
2.12\end{array}$ & $\begin{array}{c}V \\
2.12\end{array}$ & $\begin{array}{c}V \\
2.12\end{array}$ & $\begin{array}{c}V \\
2.12\end{array}$ & $\begin{array}{c}V \\
2.12\end{array}$ & $\begin{array}{c}V \\
2.12 \\
\end{array}$ & $\begin{array}{c}V \\
2.51\end{array}$ \\
\hline & & & & & & $\begin{array}{c}V \\
2.12\end{array}$ & $\begin{array}{c}V \\
2.12\end{array}$ & $\begin{array}{c}V \\
2.12\end{array}$ & $\begin{array}{c}V \\
2.12\end{array}$ & $\begin{array}{c}V \\
2.12\end{array}$ & $\begin{array}{c}V \\
2.51\end{array}$ \\
\hline & & & & & & & $\begin{array}{c}V \\
2.12 \\
\end{array}$ & $\begin{array}{c}V \\
2.12 \\
\end{array}$ & $\begin{array}{c}V \\
2.12 \\
\end{array}$ & $\begin{array}{c} \\
2.12 \\
\end{array}$ & $\begin{array}{c}V \\
2.51 \\
\end{array}$ \\
\hline & & & & & & & & $\begin{array}{c}V \\
2.12\end{array}$ & $\begin{array}{c}V \\
2.12 \\
\end{array}$ & $\begin{array}{c}V \\
2.12 \\
\end{array}$ & $\begin{array}{c}V \\
2.51 \\
\end{array}$ \\
\hline & & & & & & & & & $\begin{array}{c}V \\
2.12\end{array}$ & $\begin{array}{c}V \\
2.12\end{array}$ & $\begin{array}{c}V \\
2.51\end{array}$ \\
\hline & & & & & & & & & & $\begin{array}{c}V \\
2.12 \\
\end{array}$ & $\begin{array}{c}V \\
2.51 \\
\end{array}$ \\
\hline & & & & & & & & & & & $\begin{array}{l}V \\
2.51 \\
\end{array}$ \\
\hline
\end{tabular}


mainder can be obtained by reflection on the diagonal. We note that System $I$ is a closed system under multiplication. Also we recall that with this table it is possible to make a corresponding table of division theorems by using 1.52 .

\section{A GENERAL MULTIPLICATION THEOREM}

3.1. On classes $(P-Q) \times(R-S)$. In the preceding part, we made a study of the classes $P \times Q$, where $P$ and $Q$ are any two classes chosen from System I. We noted that the theorems of that part gave rise to theorems relative to the class $P \times Q$ if $P$ and $Q$ are both in System II, because of the fact that if $P$ is in I, then $P_{-1}$ is in II, and from $P \times Q=R$ it follows that $P_{-1} \times Q_{-1}=R_{-1}$. A natural question is to characterize $P \times Q$ if $P$ is in System I and $Q$ in System II. The answer to this happens to be trivial. For we have:

3.11. Theorem. If $P$ is in System I and $Q$ in System II, then $P \times Q=V$, unless $P$ or $Q$ is the null class 0 , in which case $P \times Q=0$.

To prove this theorem, it is obviously sufficient to consider only the case in which $P=C_{0}^{\prime \prime}$ and $Q=C_{-0}^{\prime \prime}$. Further since $P \times Q$ is contained in $V$ for any $P$ and $Q$, we need only show that if $\gamma=\left\{c_{n}\right\}$ is any sequence of $V$, then there exist $\alpha=\left\{a_{n}\right\}$ of $C_{0}^{\prime \prime}$ and $\beta=\left\{b_{n}\right\}$ of $C_{-0}^{\prime \prime}$, such that $c_{n}=a_{n} b_{n}$. We note that if $\alpha$ is any sequence of $C_{0}^{\prime \prime}$ and if we define $\beta=\gamma \times \alpha^{-1}$ then $\beta$ will be of $C_{-0}^{\prime \prime}$, provided $\gamma$ is bounded from zero, that is, in $F_{-1}$. Also if $\beta$ is of $C_{-0}^{\prime \prime}$ and $\alpha=\gamma \times \beta^{-1}$, then $\alpha$ is of $C_{0}^{\prime}$ if $\gamma$ is bounded from infinity, that is, in $F$. Assume then that $\gamma$ is in $-F_{-1} \cap-F$. Then divide the sequence of integers $n$ into two mutually exclusive ordered sets $n_{k}^{\prime}$ for which $c_{n} \geqq 1$ and $n_{k}^{\prime \prime}$ for which $c_{n}<1$. Let $\alpha_{0}$ be any element of $C_{0}^{\prime \prime}$ and $\beta_{0}$ any element of $C_{-0}^{\prime \prime}$. Define $\alpha=\left\{a_{n}\right\}$ as follows:

$$
a_{n}=a_{0 k} \quad \text { if } \quad n=n_{k}^{\prime}, \quad a_{n}=c_{n} / b_{0 k} \quad \text { if } \quad n=n_{k}^{\prime \prime} ;
$$

so that

$$
b_{n}=c_{n} / a_{0 k} \quad \text { if } \quad n=n_{k}^{\prime}, \quad b_{n}=b_{0 k} \quad \text { if } \quad n=n_{k}^{\prime \prime} .
$$

Then obviously $\alpha$ is of $C_{0}^{\prime \prime}$ and $\beta$ is of $C_{-0}^{\prime \prime}$.

The System I (as well as II) defines also classes by taking differences, particularly the class $P-Q$ where $P$ properly contains $Q$. Since if $P$ is in $I$ then $-Q$ is in II, and conversely, the classes $P-Q$ are really products of a class in I by one in II. There arises the question of the characterization of the classes $(P-Q) \times(R-S)$. If we note that $P-Q$ is in fact $P \cap-Q$, then if a product theorem similar to 1.51 were universally applicable, we might guess that 
(M): $\quad(P-Q) \times(R-S)=(P \times R) \cap(-Q \times-S)$.

If $Q=S=0$, or $P=R=V$, the identity is trivial. If we take $Q=0$, $R=V$ then the guess would give

$$
P \times-S=(P \times V) \cap(-0 \times-S)=V
$$

since $P \neq 0$ and $S \neq V$. If $P$ and $S$ are in I, then $-S$ is in II and this is Theorem 3.11. The object of this part is to determine limitations on the classes $P, Q, R$ and $S$ so that the equality (M) is valid in System I. Corresponding results for System II follow. Throughout this part it will be assumed that $P$ properly contains $Q$ and $R$ properly contains $S$ in the differences $P-Q, R-S$.

The method of procedure will be to find conditions for the validity of the equations

$$
(P-Q) \times R=P \times R \text { and }(P-Q) \times-S=-Q \times-S,
$$

which are the special cases of the general equation for $Q=0$ and $R=V$, respectively, and from these to derive the general equality.

It is however not necessary to prove both of these two equalities, since we have:

3.12. TheOREM. A theorem of the form $(P-Q) \times R=P \times R$ has as consequence a theorem of the form $\left(P^{\prime}-Q^{\prime}\right) \times-S^{\prime}=-Q^{\prime} \times-S^{\prime}$, and conversely.

For if $(P-Q) \times R=P \times R$, then $\left(P_{-1}-Q_{-1}\right) \times R_{-1}=P_{-1} \times R_{-1}$. Now if $P$ is in $\mathrm{I}$, then $P_{-1}$ is in II, and $-P_{-1}$ is a member of I. If we set $P^{\prime}=-Q_{-1}, Q^{\prime}=-P_{-1}, S^{\prime}=-R_{-1}$, then obviously $Q^{\prime}$ is properly contained in $P^{\prime}$ and our identity becomes

$$
\left(P^{\prime}-Q^{\prime}\right) \times-S^{\prime}=-Q^{\prime} \times-S^{\prime}
$$

Because of the dual nature of these two equalities, we shall refer to the process of substitution involved as a dualizing process.

We might also note that the Systems I and II are combinations of half of the System I, namely,

$$
0, C_{0}^{\prime \prime}, C_{e}^{\prime}, C_{e}, C_{e}^{\prime \prime}, C_{\infty}^{\prime}, L_{0}, F \cap-F_{-1}, F \text {. }
$$

If we denote this set of classes by Ia, the balance by Ib, and similarly for II, then

$$
3.13 \mathrm{Ib}=-(\mathrm{Ia})_{-1}, \quad \mathrm{II} \mathrm{a}=(\mathrm{Ia})_{-1}, \quad \text { and } \mathrm{IIb}=(\mathrm{Ib})_{-1}=-\mathrm{Ia} \text {, }
$$

so that

$$
I=(I a, I b)=\left(I a,-(I a)_{-1}\right) \text { and } I I=\left((I a)_{-1},-I a\right) .
$$


3.2. On the relation $(P-Q) \times R=P \times R$. It is obvious that if $Q$ is properly contained in $P$, then $(P-Q) \times R$ is contained in $P \times R$. Hence we need to derive only sufficient conditions so that $(P-Q) \times R$ contains $P \times R$. In order to state a general theorem easily, we define a dominance property related to two classes, namely:

3.21. Definition. The pair of classes $P, Q$ with $P$ properly containing $Q$ have the dominance property $K_{2}$ relative to each other, if for any element $\beta$ of $Q$ there exists an element $\alpha$ of $P-Q$ which ultimately dominates $\beta$, that is, $a_{n} \geqq b_{n}$ for $n>n_{0}$.

Also dually:

3.211. Definition. The pair of classes $P, Q$ with $P$ properly containing $Q$ have the dominance property $\bar{K}_{2}$ relative to each other, if for every $\alpha$ of $-P$, there exists a $\beta$ of $P-Q$ such that $\beta$ is ultimately dominated by $\alpha$.

We then have the following theorem.

3.22. TheOREM. If $R$ has the dominance property $\Delta_{0}^{\prime \prime}$, and $P$ and $Q$ have the dominance property $K_{2}$ with respect to each other, then $(P-Q) \times R \supset P \times R$, and so $(P-Q) \times R=P \times R$.

The proof of this is almost self-evident. Suppose $\delta$ is of $P \times R$, that is, there exists $\alpha$ of $P$ and $\gamma$ of $R$ such that $\delta=\alpha \times \gamma$. If $\alpha$ is of $P-Q$, no further steps are necessary. If however $\alpha$ is of $Q$ let $\beta$ be of $P-Q$ ultimately dominating $\alpha$ and set $\gamma_{1}=\delta \times \beta^{-1}=\alpha \times \gamma \times \beta^{-1}$. Then $\gamma_{1}$ is ultimately dominated by $\gamma$ and so of $R$, that is, $\delta=\beta \times \gamma_{1}$ is of $(P-Q) \times R$.

In order to apply this theorem to the case where $P, Q$ and $R$ are classes in System I, we show:

3.23. If $P$ and $Q$ are any two classes of System I, $P$ properly containing $Q$, and excluding the case where $P$ is $F \cup-F_{-1}$, then $P$ and $Q$ have the dominance property $K_{2}$ relative to each other.

The method of demonstrating this property for any two classes of System I chosen from Ia, that is, those lying between 0 and $F$ inclusive, is illustrated by the following special case:

3.231. $P=C_{\theta}, Q=C_{\theta}^{\prime}$. Let $\alpha$ be any element of $C_{\theta}^{\prime}$. Then since by 1.4, $C_{\theta}$ properly contains $C_{\theta}^{\prime}$, there exists an element $\beta_{0}$ of $C_{\theta}-C_{\theta}^{\prime}$. Define $\beta$ so that $b_{n}=b_{0 n}$ if $a_{n} \leqq b_{0 n}$, and $b_{n}=a_{n}$ if $a_{n} \geqq b_{0 n}$. Then since $\beta \geqq \beta_{0}$, it follows that $\beta$ is not of $C_{a}^{\prime}$. On the other hand since

$$
\sum_{n} b_{n}^{e}=\sum_{n^{\prime}} b_{0 n^{\prime}}^{e}+\sum_{n^{\prime \prime}} a_{n}^{e}<\sum b_{0 n}^{e}+\sum a_{n}^{e}
$$

it follows that $\beta$ is in $C_{\theta}$. 
It is obvious that in this proof we used the fact that $-Q$ has the property $\Delta_{0}^{\prime}$ in reverse, which is true for all $Q$ in System I. Further we have used the fact that if $\alpha$ and $\beta$ are sequences of $P$, then $\gamma$, the greater of $\alpha$ and $\beta$, is also in $P$. This holds for all of the classes of System Ia, excepting $F \cap-F_{-1}$. However the procedure above is still effective in this case, since if $\beta$ is of $Q$ and $Q$ is properly contained in $F \cap-F_{-1}$, then $\alpha$ is of $L_{0}$. If $\beta$ is of $F \cap-F_{-1}$, and $\alpha$ is of $L_{0}$, then the greater of $\alpha$ and $\beta$ is still in $F \cap-F_{-1}$. Hence we have the theorem:

3.232. Theorem. Any two classes $P, Q$ with $P$ properly containing $Q$ from System Ia have the property $K_{2}$ relative to each other.

This is no longer true if we continue in the System I; we have:

3.233. The class $F \cup-F_{-1}$ does not have the property $K_{2}$ relative to $-F_{-1}$, or relative to $F$, but it does have the property $K_{2}$ relative to $F \cap-F_{-1}$, and so relative to all the other classes of System $\mathrm{I}$.

Let $\alpha$ be an element of $-F_{-1}$ for which both $\lim \sup a_{n}=\infty$, and $\lim$ inf $a_{n}=0$. Then there exists in $F \cup-F_{-1}$ no dominating element belonging to $-\left(-F_{-1}\right)$, that is, $F_{-1}$. Similarly if $\alpha$ is in $F$ with $\lim \inf a_{n}>0$, there does not exist in $\left(F \cup-F_{-1}\right)-F$, that is, in $-F_{-1} \cap-F$, an element dominating $\alpha$. On the other hand let $\alpha$ belong to $F \cap-F_{-1}$, and let $\lim _{k} a_{n_{k}}=0$, with $a_{n_{k}} \leqq 1$. Define $\beta=\left\{b_{n}\right\}$ by the conditions $b_{n}=a_{n}$ for $n \neq n_{k}$, or for $n=n_{k}, k$ even, and $b_{n}=1 / a_{n}$ with $n=n_{l}, k$ odd. Then $\beta$ is of $-F_{-1}$, dominates $\alpha$, but is not in $F \cap-F_{-1}$.

For the remaining members of System I, we demonstrate the property $K_{2}$ between adjacent classes, from which it then follows in general. We consider the following cases:

3.234. $-L_{\infty}$ has the property $K_{2}$ relative to $-F_{-1}, F$, and $F \cup-F_{-1}$, and consequently all classes of System I.

Suppose that $\alpha$ belongs to $F$ and $\lim \inf a_{n}>0$, that is, $\alpha$ is not in $-F_{-1}$. Then define $\beta: b_{n}=a_{n}$ for $n$ even, $b_{n}=n a_{n}$ for $n$ odd. Then $\beta$ is in $-L_{\infty}$, but not in $F$ or $-F_{-1}$.

Suppose $\alpha$ belongs to $-F_{-1}$. Let $n^{\prime}$ be the set of integers for which $a_{n} \leqq 1$. Divide the integers $n^{\prime}$ into two infinite subsets $n^{\prime \prime}, n^{\prime \prime \prime}$ so that $\lim _{n^{\prime \prime}} a_{n^{\prime \prime}}=0$. Then define $b_{n}=a_{n}$ for $n \neq n^{\prime}, b_{n}=1 / a_{n}$ for $n=n^{\prime \prime}$ and $b_{n}=1$ for $n=n^{\prime \prime \prime}$. Then $\beta$ is of $-L_{\infty}$, but not in $F$ nor $-F_{-1}$.

3.235. $D_{-\infty}^{\prime \prime}$ has the $K_{2}$ property relative to $-L_{\infty}$.

Let $\alpha$ be of $-L_{\infty}$, that is, lim inf $a_{n}=a_{0}<\infty$ and suppose that lim $a_{n_{k}}=a_{0}$. It is obviously sufficient to assume $a_{0}>0$. Suppose $\beta_{0}$ is any element of $D_{-\infty}^{\prime \prime}$ not belonging to $-L_{\infty}$, for example, $n \exp \left(1 /(\log n)^{1 / 2}\right)$. Define $\beta=\left\{b_{n}\right\}$ by the conditions $b_{n}=n a_{n}$ for $n \neq n_{k}, b_{n}=b_{0 k} a_{n}$ for $n=n_{k}$. Then $\beta$ is in $D_{-\infty}^{\prime \prime}$ but $\lim _{n} b_{n}=\infty$. 
3.236. All pairs of classes $P, Q$ in the $D$ group of System I have the $K_{2}$ property relative to each other.

We prove this by showing that the theorem holds for the following cases: (a) $D_{-e}, D_{-f}$ with $e<f$, (b) $D_{-1}, D_{-1}^{\prime}$, and (c) $D_{-1}^{\prime \prime}, D_{-1}$, since obviously the case of any two pairs from the $D$ group can be deduced from these three cases.

(a) Suppose that $\alpha$ is of $D_{-f}$, that is, $\sum_{n} a_{n}^{-f}=\infty$. Without loss of generality it is possible to assume that $a_{n} \geqq 1$. Let $\sigma=\left\{s_{n}\right\}=\left\{\sum_{1}^{n} a_{m}^{-f}\right\}$ and let $\beta=\alpha \times \sigma^{1 / e}$. Then since $\beta^{-f}=\alpha^{-f} \times \sigma^{-f / e}$ and $f / e>1$ the AbelDini Theorem assures us that $\beta^{-f}$ is of $C_{1}$, that is, $\beta$ belongs to $C_{-f}$. On the other hand

$$
\beta^{-\bullet}=\alpha^{-\bullet} \times \sigma^{-1} \geqq \alpha^{-f} \times \sigma^{-1}
$$

since $f>e$, and $a_{n} \geqq 1$. Now $\alpha^{-f} \times \sigma^{-1}$ is in $D_{1}$, that is, $\beta$ is of $D_{-e}$. Obviously $\beta$ dominates $\alpha$.

(b) Suppose $\alpha$ is in $D_{-1}^{\prime}$, that is, there exists an $f>1$, so that $\alpha$ is of $D_{-f}$. Using the same notation as in (a), we now define $\beta=\alpha \times \sigma$. Then if $g>1, \beta^{-0}=\alpha^{-0} \times \sigma^{-0} \leqq \alpha^{-1} \times \sigma^{-\sigma}$ is of $C_{1}$, that is, $\beta$ is in $C_{-o}$ for all $g>1$, and so in $C_{-1}^{\prime \prime}=-D_{-1}^{\prime}$. On the other hand $\beta$ is in $D_{-1}$ and dominates $\alpha$.

(c) Suppose $\alpha$ is in $D_{-1}$. Using the same notation as in (a) with $f=1$ and assuming $a_{n} \geqq 1$, we set $b_{n}=a_{n} s_{n} \log ^{2} s_{n}$, that is, $\beta=\alpha \times \sigma \times \log ^{2} \sigma$. Then by an extension of the Abel-Dini Theorem, ${ }^{9} \beta$ is of $C_{-1}$. On the other hand $\beta$ is of $D_{-f}$, for $f<1$. For if $f<g<1$

$$
\beta^{-f}=\alpha^{-f} \times \sigma^{-f} \times \log ^{-2 f} \sigma \geqq \alpha^{-1} \times \sigma^{-f} \times \log ^{-2 f} \sigma \geqq \alpha^{-1} \times \sigma^{-\sigma}
$$

ultimately, since $\lim _{n} s_{n}^{\theta-\rho} \log ^{-2 f} s_{n}=\infty$.

The fact that $V$ has the $K_{2}$ property with respect to any class in System I (or System II) is trivial.

With respect to the property $\bar{K}_{2}$, we have:

3.237. If $P$ and $Q$ are any two classes of System I, with $P$ properly containing $Q$, and $(P, Q)$ isneither $\left(F, F \cap-F_{-1}\right)$ nor $\left(-F_{-1}, F \cap-F_{-1}\right)$, then $P$ and $Q$ have the $\bar{K}_{2}$ property relative to each other, that is, for every element $\alpha$ of $-P$, there exists an element $\beta$ of $P-Q$, such that $\alpha$ ultimately dominates $\beta$.

This follows immediately from the preceding results by the dualizing process.

As a consequence of these results, we are able to state the following theorems:

- See T. H. Hildebrandt, Remarks on the Abel-Dini Theorem, Amer. Math. Monthly vol. 49 (1942) p. 443. 
3.241. The relation $(P-Q) \times R=P \times R$ provided $P, Q$ and $R$ are classes of System I, excepting possibly $(P, Q)=\left(F \cup-F_{-1},-F_{-1}\right)$ or $\left(F \cup-F_{-1}, F\right)$.

3.242. The relation $(P-Q) \times-S=-Q \times-S$, with $P$ properly containing $Q$, holds for all classes of System $\mathrm{I}$, excepting possibly $(P, Q)$ $=\left(F, F \cap-F_{1}\right)$ or $\left(-F_{-1}, F \cap-F_{-1}\right)$.

For the exceptional cases we have:

3.243. The relation $\left(\left(F \cup-F_{-1}\right)-\left(-F_{-1}\right)\right) \times R=\left(F \cup-F_{-1}\right) \times R$ is not valid for any $R$ of System I excepting 0 and $V$.

For if $\alpha$ belongs to $\left(F \cup-F_{-1}\right)-\left(-F_{-1}\right)$, then $\infty>\lim \sup a_{n}$ $>\lim$ inf $a_{n}>0$. Consequently $\left(\left(F \cup-F_{-1}\right)-\left(-F_{-1}\right)\right) \times R=R$. But from the final table of Part II it is obvious that $\left(F \cup-F_{-1}\right) \times R=R$ only for $R=0$ and $V$.

Applying 3.12 we have:

3.244. The relation $\left(\left(F-\left(F \cap-F_{-1}\right) \times-S=-\left(F \cap-F_{-1}\right) \times-S\right.\right.$ is valid for no $S$ excepting the classes 0 and $V$ in System I.

On the other hand we have:

3.245. The relation $\left(\left(F \cup-F_{-1}\right)-F\right) \times R=\left(F \cup-F_{-1}\right) \times R$ holds for all $R$ of System I excepting $F$.

By Part II, $\left(F \cup-F_{-1}\right) \times R=-F_{-1}$, for any $R$ between $C_{0}^{\prime \prime}$ and $F \cap-F_{-1}$ inclusive. On the other hand, the class $\left(F \cup-F_{-1}\right)-F$ is the class of sequences $\alpha$ such that $\lim \sup a_{n}=\infty$ and $\lim \inf a_{n}=0$. Let $\alpha$ be any element of $-F_{-1}$ and $\beta_{0}$ any element of $C_{0}^{\prime \prime}$. Select the integers $n_{k}$ so that $c_{n_{k}}<b_{0 k}$. Let $b_{n}=b_{0 n}$ for $n \neq n_{k}, b_{n}=\left(b_{0 k}\right)^{1 / 2}$ for $n=n_{k}$ and $k$ even, $b_{n}=c_{n} / k$ for $k$ odd. Then for $k$ odd $a_{n_{k}}=c_{n} / b_{n}<\left(b_{0 k}\right)^{1 / 2}$ which approaches zero with $k$, while for $k$ even $a_{n_{k}}=k$ and $b_{n_{k}}<b_{0 k} / k$. It follows that $\beta$ is of $C_{0}^{\prime \prime}$ and $\alpha$ of $\left(F \cup-F_{-1}\right)-F$, so that $\left(\left(F \cup-F_{-1}\right)-F\right)$ $\times C_{0}^{\prime \prime} \supset-F_{-1}$. The same type of proof works for all classes $R$ between and including $C_{0}^{\prime \prime}$ and $F \cap-F_{-1}$.

For $R$ between and including $-F_{-1}$ and $V$ we have $\left(F \cup-F_{-1}\right) \times R$ $=V$. It is consequently sufficient to prove that $\left(\left(F \cup-F_{-1}\right)-F\right)$ $\times\left(-F_{-1}\right) \supset V$. Let $\gamma$ be any element of $V$ and suppose in the first place that $\lim \sup c_{n}=\infty$, that is, there exists a subsequence such that $\lim _{k} c_{n_{k}}=\infty$. For $n \neq n_{k}$, take $a_{n}$ and $b_{n}$ arbitrary with $a_{n} b_{n}=c_{n}$. For $n=n_{k}$ and $k$ even, take $a_{n}=c_{n}^{2}$, so that $b_{n}=1 / c_{n}$. For $n=n_{k}$ and $k$ odd, take $a_{n}=1 / c_{n}, b_{n}=c_{n}^{2}$. Then both $\alpha$ and $\beta$ are of $\left(F \cup-F_{-1}\right)-F$ and so also of $-F_{-1}$. Suppose next that $\infty>\lim \sup c_{n}>0$, so that there exists a sequence $c_{n_{k}}$ such that $\lim _{k} c_{n_{k}}=c_{\theta}>0$. Take $a_{n}$ and $b_{n}$ arbitrary for $n \neq n_{k}$, with $a_{n} b_{n}=c_{n}$. For $n=n_{k}, k$ even, take $a_{n}=k c_{n}$, so that $b_{n}=1 / k$, and for $n=n_{k}, k$ odd, take $a_{n}=c_{n} / k$, so that $b_{n}=k$. Then $\alpha$ and $\beta$ have the desired properties. Finally if $\lim c_{n}=0$, a disposi- 
tion similar to the first case will work.

For $R=F$ we have $\left(\left(F \cup-F_{-1}\right)-F\right) \times F=\left(F \cup-F_{-1}\right)-F$ but $\left(F \cup-F_{-1}\right) \times F=F \cup-F_{-1}$. This completes the proof of the theorem. As a corollary we have:

3.246. The relation $\left(\left(-F_{-1}\right)-\left(F \cap-F_{-1}\right)\right) \times-S=\left(-F_{-1}\right) \times-S$ holds for all $S$ of System I excepting $-F_{-1}$.

3.3. Decomposition theorems. It will be useful later to have certain results on the decomposition of sequences of a class into mutually exclusive subsequences belonging to the class. We have:

3.31. Theorem. If $\alpha$ is any element of a class $P$ of System I (or System II) then there exist two complementary infinite subsequences of $\alpha$ : $\alpha^{\prime}=\left\{a_{n^{\prime}}\right\}$ and $\alpha^{\prime \prime}=\left\{a_{n^{\prime \prime}}\right\}$ so that each subsequence belongs to $P$.

For the classes which are characterized by a convergence property, this theorem is obvious, since every subsequence of any element of such a class has the same convergence property. The same is true of the class $L_{0}$ and $F$. For an element $\alpha$ of $F \cap-F_{-1}$, we select a subsequence $\left\{a_{n_{k}}\right\}$ such that $\lim _{k} a_{n_{k}}=0$, decompose it into two sequences having the same property and include the remaining elements of the original sequence in either of these subsequences. The same procedure is obviously applicable to $-F_{-1}$, and consequently to $F \cup-F_{-1}$. For an element $\alpha$ of $-L_{\infty}$, we use a similar procedure, excepting that in this case, we utilize a subsequence $a_{n_{k}}$ such that $\lim _{k} a_{n_{k}}=a_{0}<\infty$. For the classes of divergent character, $D_{-e}$ and $D_{-\theta}^{\prime}$, the following procedure is effective. Select a subsequence $n_{k}$ such that

$$
\sum_{n_{k}-1 \leq n<n_{k}} a_{n}^{-\bullet}>1 .
$$

Take $n=n^{\prime}$ if $n_{k-1} \leqq n<n_{k}, k$ even, and $n=n^{\prime \prime}$ for $k$ odd. For the classes $D_{-e}^{\prime \prime}$ we modify this procedure slightly. Let $f_{k}$ approach $e$ monotonely decreasing, and select $n_{k}$ by the condition

$$
\sum_{n_{k}-1 \leqq n<n_{k}} a_{n}^{-f_{k}}>1 .
$$

Then the same decomposition as in the preceding case will give two subsequences each in $D_{-e}^{\prime \prime}$.

For System II the result follows from the one-to-one correspondence between sequences $\alpha$ and $\alpha^{-1}$.

For products of classes we have a similar result, namely:

3.32. TheOREM. If $P$ and $Q$ are any two classes chosen either from 
System I or II, and $\alpha$ belongs to $P \cap Q$, then there exist complementary subsequences $\left\{a_{n^{\prime}}\right\}$ and $\left\{a_{n^{\prime \prime}}\right\}$ of $\alpha$ each of which belongs to $P \cap Q$.

The proof of this theorem depends upon the following lemma valid for all classes or properties of System I and System II :

3.33. LemMa. If $\alpha_{0}$ is any sequence having the property $P$ and $\alpha_{1}$ is a subsequence of a sequence having the property $P$ then any combination of $\alpha_{0}$ and $\alpha_{1}$ into a single sequence has property $P$.

Obviously this includes the case when the sequence $\alpha_{1}$ has the property $P$.

We return to the proof of 3.32. Let $\alpha$ belong to $P \cap Q$. Then there exist $\alpha^{\prime}=\left\{a_{n^{\prime}}\right\}$ and $\alpha^{\prime \prime}=\left\{a_{n^{\prime \prime}}\right\}$, complementary subsequences of $\alpha$ each belonging to $P$. By 3.33 one of these sequences must belong to $Q$. Suppose it is the first. Then this can be divided into two subsequences $\alpha^{\prime \prime \prime}=\left\{a_{n^{\prime \prime}}\right\}$ and $\alpha^{\text {iv }}=\left\{a_{n}{ }^{\text {iv }}\right\}$ both of which belong to $Q$ and one of which belongs to $P$. Let it be the first. Then $\alpha^{\prime \prime \prime}$ and the rearrangement of $\left\{a_{n^{\prime \prime}}\right\}\left\{a_{n}{ }^{\text {iv }}\right\}$ in proper order gives by 3.33 two complementary subsequences each belonging to both $P$ and $Q$. For $\alpha^{\prime \prime}$ is of $P$ and $\alpha^{\text {iv }}$ is a subsequence of a sequence of $P$, while $\alpha^{\text {iv }}$ is of $Q$ and $\alpha^{\prime \prime}$ is a subsequence of a sequence of $Q$. As a corollary we have:

3.34. If $P$ and $Q$ are classes of System I, such that $P$ properly contains $Q$, then any sequence of $P-Q$ can be divided into two complementary subsequences both of which belong to $P-Q$.

We finally make the following two observations:

3.35. The classes $P$ of the System Ia with the exception of $F \cap-F_{-1}$ have the property: If $\alpha$ is of $P$ then every subsequence of $\alpha$ has the property $P$.

This holds also for the classes of System IIa, excepting $F_{-1} \cap-F$.

3.36. The classes $P$ of the System Ib with the exception of $F \cup-F_{-1}$ have the property: If $\alpha^{\prime}$ is a subsequence of a sequence of $P$, which has the property $P$, then the sequence $\beta$ obtained by altering in any way the elements of $\alpha$ complementary to $\alpha^{\prime}$ will have property $P$.

3.4. The multiplication theorem. We are now in position to prove the following theorem.

3.41. Multiplication Theorem. If $P, Q, R$ and $S$ are of System $\mathrm{I}$, neither $P$ nor $R$ is $F \cup-F_{-1}$, and $Q$ nor $S$ is $F \cap-F_{-1}, P$ properly contains $Q$, and $R$ properly contains $S$, then $(P-Q) \times(R-S)=(P \times R)$ $\cap(-Q \times-S)$.

As we have already noted that $(P-Q) \times(R-S) \subset(P \times R)$ 
$\cap(-Q \times-S)$, only the inverse inclusion needs to be shown. Since neither $P$ nor $R$ are $F \cup-F_{-1}$ and $Q$ nor $S$ are $F \cap-F_{-1}$, the application of Theorems 3.241 and 3.242 yields

$$
\begin{aligned}
P \times R & =(P-Q) \times R=P \times(R-S), \\
-Q \times-S & =(P-Q) \times-S=-Q \times(R-S) .
\end{aligned}
$$

If then $\gamma$ is a member of $(P \times R) \cap(-Q \times-S)$, it also belongs to the four classes $(P-Q) \times R,(P-Q) \times-S, P \times(R-S),-Q \times(R-S)$, that is, we can write

$$
\gamma=\alpha_{1} \times \beta_{1}=\alpha_{2} \times \beta_{2}=\alpha_{3} \times \beta_{3}=\alpha_{4} \times \beta_{4}
$$

where $\alpha_{1}$ and $\alpha_{2}$ are of $P-Q, \alpha_{3}$ is of $P, \alpha_{4}$ is of $-Q, \beta_{1}$ is of $R, \beta_{2}$ is of $-S$, and $\beta_{3}$ and $\beta_{4}$ are of $R-S$. Obviously the theorem is proved unless $\beta_{1}$ is of $S, \beta_{2}$ is of $-R, \alpha_{3}$ is of $Q$ and $\alpha_{4}$ is of $-P$. We shall consequently make these assumptions. We break up the proof in to cases.

Case I. $P$ and $R$ are both of System Ib with $F \cup-F_{-1}$ omitted. We assume then

$$
\begin{aligned}
& \gamma=\alpha_{2} \times \beta_{2} \text { with } \alpha_{2} \text { of } P-Q, \beta_{2} \text { of }-R, \\
& \gamma=\alpha_{4} \times \beta_{4} \text { with } \alpha_{4} \text { of }-P, \quad \beta_{4} \text { of } R-S .
\end{aligned}
$$

By 3.34, the sequence $\alpha_{2}$ can be divided into two subsequences, $\alpha_{2}^{\prime}$ and $\alpha_{2}^{\prime \prime}$, such that both are in $P-Q$. Consider the corresponding sequences $\beta_{4}^{\prime}$ and $\beta_{4}^{\prime \prime}$. Since $\beta_{4}$ is of $R$, by 3.33 , not both $\beta_{4}^{\prime}$ and $\beta_{4}^{\prime \prime}$ can be of $-R$, and since $\beta_{4}$ is of $-S$ not both $\beta_{4}^{\prime}$ and $\beta_{4}^{\prime \prime}$ can be of $S$. By considering all possibilities it develops that we have either $\beta_{4}^{\prime}$ or $\beta_{4}^{\prime \prime}$ is of $R-S$, or one is of $-R$ and the other of $S$.

Suppose then that $\beta_{4}^{\prime}$ is of $R-S$. Then we define $\alpha=\left(\alpha_{4}^{\prime}, \alpha_{2}^{\prime \prime}\right)$, $\beta=\left(\beta_{4}^{\prime}, \beta_{2}^{\prime \prime}\right)$ arranged in proper order. Then since $\alpha_{2}^{\prime \prime}$ is of $P-Q$, and $P$ is in Ib, and not $F \cup-F_{-1}, \alpha$ is of $P$ by 3.36. Since $\alpha_{2}^{\prime \prime}$ is of $-Q$, and $\alpha_{4}^{\prime}$ is a subsequence of $\alpha_{4}$ which is of $-P$ and so of $-Q, \alpha$ is of $-Q$ by 3.33. A repetition of this reasoning shows that $\beta$ is of $R-S$.

In case one of the $\beta_{4}^{\prime}, \beta_{4}^{\prime \prime}$ is of $-R$ and the other of $S$ suppose that $\beta_{4}^{\prime}$ is of $-R$. Then by 3.237 there exists a sequence $\beta_{0}^{\prime} \leqq \beta_{4}^{\prime}$ of $R-S$, if $R$ is in the $\bar{K}_{2}$ relation to $S$, which is true since $S$ is not the class $F \cap-F_{-1}$. Define $\alpha=\left(\alpha_{4}^{\prime} \times \beta_{4}^{\prime} \times \beta_{0}^{\prime-1}, \alpha_{2}^{\prime \prime}\right)$ and $\beta=\left(\beta_{0}^{\prime}, \beta_{2}^{\prime \prime}\right)$. Then $\beta$ is of $R-S$ as above. Further $\alpha$ is of $P$ as above. On the other hand $\alpha^{\prime} \geqq \alpha_{2}^{\prime}$, and consequently is a subsequence of a sequence having the property $-P$ and so of $-Q$. Hence $\alpha$ is of $-Q$.

Case II. $P$ is in Ia, $R$ in Ib with $F \cup-F_{-1}$ omitted. Then take

$$
\begin{aligned}
& \gamma=\alpha_{2} \times \beta_{2} \text { with } \alpha_{2} \text { of } P-Q, \beta_{2} \text { of }-R, \\
& \gamma=\alpha_{3} \times \beta_{3} \text { with } \alpha_{3} \text { of } Q, \beta_{3} \text { of } R-S .
\end{aligned}
$$


Proceed as in Case I, that is, break up $\alpha_{2}=\left(\alpha_{2}^{\prime}, \alpha_{2}^{\prime \prime}\right)$ each of $P-Q$ and assume in the first place that $\beta_{3}^{\prime}$ is of $R-S$. Then define $\alpha=\left(\alpha_{3}^{\prime}, \alpha_{2}^{\prime \prime}\right)$ and $\beta=\left(\beta_{3}^{\prime}, \beta_{2}^{\prime \prime}\right)$. Then $\alpha$ is of $P$ since $\alpha_{2}^{\prime \prime}$ has property $P$ and $\alpha_{3}^{\prime}$ is a subsequence of a sequence of $P . \alpha_{2}^{\prime \prime}$ is of $-Q$. Then any sequence added to $\alpha_{2}^{\prime \prime}$ will give a sequence which is still in $-Q$, if $Q$ is in Ia and not $F \cap-F_{-1}$. Then $\alpha$ is of $-Q$. The proof that $\beta$ is of $R-S$ is the same as in Case I above.

If one of the pair $\beta_{3}^{\prime}, \beta_{3}^{\prime \prime}$ is of $-R$ and the other of $S$, we assume $\beta_{3}^{\prime}$ of $S$. Then there exists a $\beta_{0}^{\prime} \geqq \beta_{3}^{\prime}$ of $R-S$, since $R$ has the $K_{2}$ property relative to $S$, when $R$ is not $F \cup-F_{-1}$. Let $\alpha=\left(\alpha_{8}^{\prime} \times \beta_{3}^{\prime} \times \beta_{0}^{\prime-1}, \alpha_{2}^{\prime \prime}\right)$, $\beta=\left(\beta_{0}^{\prime}, \beta_{2}^{\prime \prime}\right)$. Obviously $\beta$ is of $R-S$. On the other hand since $\alpha^{\prime}=\alpha_{3}^{\prime} \times \beta_{3}^{\prime} \times \beta_{0}^{\prime-1} \leqq \alpha_{3}^{\prime}$, and $\alpha_{3}^{\prime}$ is of $Q$, it follows that $\alpha$ is of $P$. Further $\alpha$ is of $-Q$ as above.

Case III. $P$ is in Ib with $F \cup-F_{-1}$ omitted, $R$ in Ia. This follows by parity from Case II.

Case IV. $P$ is in Ia, $R$ in Ia. This can be deduced from Case I by the dualization process. We can also proceed directly. We take

$$
\begin{aligned}
& \gamma=\alpha_{1} \times \beta_{1} \text { with } \alpha_{1} \text { of } P-Q, \beta_{1} \text { of } S, \\
& \gamma=\alpha_{3} \times \beta_{3} \text { with } \alpha_{3} \text { of } Q, \beta_{3} \text { of } R-S,
\end{aligned}
$$

and follow Case II.

This concludes the proof of the theorem. We might point out that the reasoning in Case II still applies if $P$ is any class in System I, $Q$ is in Ia with $F \cap-F_{-1}$ omitted, $R$ in Ib with $F \cup-F_{-1}$ omitted, and $S$ any class in I; while Case IV applies if $P$ and $R$ are in System I, $Q$ and $S$ are in System Ia with $F \cap-F_{-1}$ omitted, and $R$ has the $K_{2}$ relation to $S$. By a careful analysis of the sufficient conditions involved, it is possible to extend the multiplication theorem to cases in which $P$ or $R$ are the class $F \cup-F_{-1}$, and $Q$ or $S$ the class $F \cap-F_{-1}$. A complete analysis requires the use of the Table of Part II and is rather tedious. We therefore conclude with a combination of classes where the multiplication theorem is not valid, namely:

3.42. Theorem. If $P$ is the class $F \cup-F_{-1}$, and $Q$ is the class $-F_{-1}$, then for no $R$ properly containing $S$ is $(P-Q) \times(R-S)=(P \times R)$ $\cap(-Q \times-S)$; and dually the equality does not hold if $Q$ is the class $F \cap-F_{-1}$ and $P$ the class $F, R$ and $S$ any.

This follows at once from a reference to the table of Part II. 